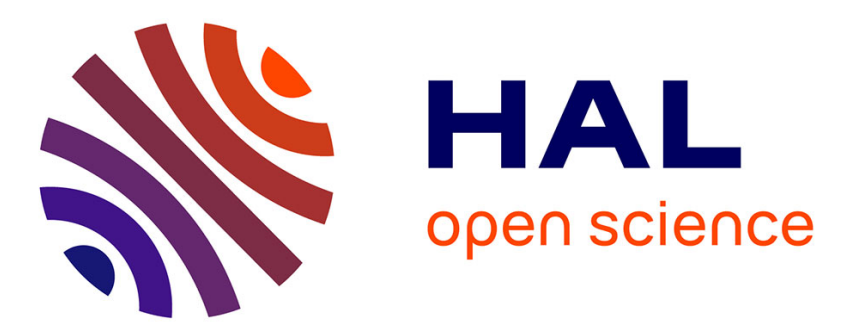

\title{
Using staggered grids with characteristic boundary conditions when solving compressible reactive Navier-Stokes equations
}

\author{
Eric Albin, Yves d'Angelo, Luc Vervisch
}

\section{- To cite this version:}

Eric Albin, Yves d'Angelo, Luc Vervisch. Using staggered grids with characteristic boundary conditions when solving compressible reactive Navier-Stokes equations. International Journal for Numerical Methods in Fluids, 2010, 68 (05), pp.546-563. 10.1002/fld.2520 . hal-00967383

\section{HAL Id: hal-00967383 https://hal.science/hal-00967383}

Submitted on 28 Mar 2014

HAL is a multi-disciplinary open access archive for the deposit and dissemination of scientific research documents, whether they are published or not. The documents may come from teaching and research institutions in France or abroad, or from public or private research centers.
L'archive ouverte pluridisciplinaire HAL, est destinée au dépôt et à la diffusion de documents scientifiques de niveau recherche, publiés ou non, émanant des établissements d'enseignement et de recherche français ou étrangers, des laboratoires publics ou privés. 


\title{
Using staggered grids with characteristic boundary conditions when solving compressible reactive Navier-Stokes equations
}

\author{
Albin $^{1}$, Yves D'Angelo ${ }^{1}$, Luc Vervisch ${ }^{1}$ \\ ${ }^{1}$ CORIA, CNRS \& INSA de Rouen, Campus du Madrillet, \\ 76801 Saint-Etienne du Rouvray cedex, FRANCE.
}

\begin{abstract}
A specific (hybrid) arrangement of variables is discussed to solve reactive compressible Navier-Stokes equations on staggered-like grids with high order finite difference schemes. The objective is to improve the numerical flow solution at boundaries. Hybrid arrangement behavior is compared to 'pure' colocated and staggered strategies. Classical Fourier analysis shows accuracy to be significantly improved in the hybrid case. One-dimensional laminar flame test demonstrates increased robustness (in term of mesh resolution), while computation of 1D exiting pressure wave propagation gives evidence that the method also improves accuracy in the prediction of non reflecting outflows, compared e.g. to the fully staggered scheme of [9]. Multidimensional extension is illustrated through turbulent 2D planar and 3D expanding flames simulations.
\end{abstract}

\section{INTRODUCTION}

When computing compressible reactive flows, numerical discretization schemes using staggered grid arrangements are known to be usually more stable than colocated ones [1]. The idea of using several staggered grids was introduced in [2], with differentiations and interpolations up to second order by using finite difference schemes for solving constant density flow dynamics. The major outcome was better numerical stability, with lower levels of numerical artificial viscosity. High order compact Padé schemes were later used and analyzed by Lele [6]. Nagarajan et al. [9] used compact staggered schemes for Large-Eddy Simulation (LES) of compressible subsonic flows, up to sixth-order accuracy. Boersma et al. $[10,11]$ then extended these staggered schemes up to twelfth order to simulate a turbulent burning subsonic jet.

On the other hand, numerical boundary condition treatment for compressible flows [4] has also been continuously evolving, since compressible solutions are strongly dependent on boundary conditions. Poinsot et al. [12] proposed a systematic Navier-Stokes Characterisctic treatment for acoustic Boundary Conditions, so called NSCBC. Yoo et al. [13] then showed that transverse, diffusive and source terms should no longer be neglected to specify incoming waves. Lodato et al. [14] have extended NSCBC to three-dimensional flows with edges and corners, including transverse terms at boundaries. Recently, Albin et al. [27] proposed a strategy to handle transverse outflows.

However, in such staggered grid arrangements, discrete boundaries may not be defined in a satisfying unambiguous manner, simply because several grids may coexist at computational domain boundaries. Defining a specific boundary condition may become a quite tedious task, with numerous options that may be flow configuration dependent $[9,10,11]$. This is not the case for colocated methods, where boundary points are, obviously, univocally defined. To keep the advantages of both grid arrangements, namely the increased accuracy and robustness of staggered methods and the unambiguous definition of boundary points of colocated methods, a specific boundary grid 
arrangement is proposed, in which an extra point is simply added to the staggered grid. The onesided extrapolation usually introduced at boundary $[9,10]$ is then replaced by an exact interpolation, improving accuracy not only at the staggered-grid boundary, but also, as it shall be demonstrated below, in the whole domain. This grid topology and procedure can be seen as an 'hybrid colocatedstaggered' strategy [26]. Acoustic boundary condition treatments [13, 14] are then applied in a natural manner, despite the staggered character of the grid. Note that very recent independent work by Topalian et al. [25] presents quite similar ideas. However, employed characteristic boundary treatment is different [13] and in present work stress is laid upon reactive cases. In [25], authors increase formal order accuracy at boundaries by increasing the stencil width. Here, the stencil width is kept constant. We mainly highlight the advantage of the hybrid approach when using less resolved grids.

The paper is organized as follow. Section 1 briefly presents the set of balance equations used to simulate the fully compressible reactive flow. It also describes the hybrid grid arrangement of conservative variables with the extra grid points at boundaries. Differentiation and interpolation rules needed to solve Navier-Stokes equations are introduced, with adapted notations. Implicit (Padé) and explicit hybrid finite difference schemes are presented section 2. The adopted methodology to compute finite difference formulæ at boundaries is precised in the Appendix. Section 2.2 analyses and compares transfer functions of colocated, staggered and hybrid schemes, highlighting the resulting increased accuracy for hybrid schemes. Section 3, the procedure for solving NS equations (making use of previously introduced differentiation and interpolation operators) is presented in some details. Section 4 compares the hybrid arrangement with colocated and staggered arrangements when computing a propagating pressure wave, as in [9]. Comparisons are extended through a one-dimensional laminar flame propagation (section 5). Direct extension to multi-dimensional reactive flow problems, like turbulent $2 \mathrm{D}$ planar and $3 \mathrm{D}$ expanding flames, is also proved possible. Concluding remarks finally end the paper section 6 .

\section{GOVERNING EQUATIONS, HYBRID ARRANGEMENT}

\subsection{Conservative form of NS equations}

Multi-component Navier-Stokes equations are solved in their fully compressible conservative form. They read :

$$
\begin{array}{ll}
\frac{\partial \rho}{\partial t}+\frac{\partial \rho U_{j}}{\partial x_{j}} & =0 \\
\frac{\partial \rho U_{i}}{\partial t}+\frac{\partial \rho U_{i} U_{j}}{\partial x_{j}}+\frac{\partial P}{\partial x_{i}} & =\frac{\partial \tau_{i j}}{\partial x_{j}} \\
\frac{\partial \rho E}{\partial t}+\frac{\partial(P+\rho E) U_{j}}{\partial x_{j}} & =\frac{\partial q_{E}^{j}}{\partial x_{j}}+\frac{\partial \tau_{i j} U_{i}}{\partial x_{j}}+S_{E} \\
\frac{\partial \rho Y_{k}}{\partial t}+\frac{\partial \rho Y_{k} U_{j}}{\partial x_{j}} & =\frac{\partial q_{j}^{k}}{\partial x_{j}} \quad+S_{k}
\end{array}
$$

Density is $\rho$, pressure $P$ and mass fractions $Y_{k}, k \in \llbracket 1, N_{s} \rrbracket$ ( $N_{s}$ denotes the number of species); $\rho U_{i}$ are momentum components (for $\left.i \in \llbracket 1, N_{d i m} \rrbracket\right)$. To close the system, using usual notations, it is assumed that $\tau_{i j}=\mu\left(\partial U_{i} / \partial x_{j}+\partial U_{j} / \partial x_{i}\right)-(2 / 3) \mu \operatorname{div} \mathbf{U} \delta_{i j} ; \quad \mu=\mu_{o}\left(T / T_{o}\right)^{0.76} ; \quad \rho E=$ 
$\rho C_{V} T+\rho U_{i}^{2} / 2 ; \quad q_{E}^{j}=\lambda \partial T / \partial x_{j} ; q_{k}^{j}=D \partial Y_{k} / \partial x_{j} ; \lambda=\mu C_{p} / \operatorname{Pr}$ and $D=\mu / S c_{k}$. Pressure is computed from perfect gas law $P=\rho r T ; S_{k}$ and $S_{E}$ represent source terms, e.g. combustion. In the tests presented below (see section 5), single step chemical reaction is assumed $(F+O \longrightarrow P)$. Pre-exponential factor of the Arrhenius law is a function of local equivalence ratio to fit correct value of laminar flame velocity [5].

At the boundaries, convective terms and pressure gradient components are computed by using a characteristic decomposition of the flow [13, 14, 27] (see system (2) and (3)):

$$
\begin{aligned}
\frac{\partial \rho U_{j}}{\partial x_{j}} & =d_{4}^{j} \\
\frac{\left(\partial \rho U_{i} U_{j}+P \delta_{i j}\right)}{\partial x_{j}} & =U_{i} d_{4}^{j}+\rho d_{i}^{j} \\
\frac{\partial(P+\rho E) U_{j}}{\partial x_{j}} & =\frac{1}{2} U_{k}^{2} d_{4}^{j}+\rho U_{j} d_{i}^{j}+\frac{d_{5}^{j}}{\gamma-1} \\
\frac{\partial \rho U_{j} Y_{k}}{\partial x_{j}} & =Y_{k} d_{1}+\rho d_{k}
\end{aligned}
$$

with

$$
\begin{aligned}
d_{i=j}^{j} & =\frac{\mathcal{L}_{5}^{j}-\mathcal{L}_{4}^{j}}{2 \rho c} \\
d_{i \neq j}^{j} & =\mathcal{L}_{i}^{j} \\
d_{4}^{j} & =\frac{\mathcal{L}_{j}^{j}+d_{5}^{j}}{c^{2}} \\
d_{5}^{j} & =\frac{\mathcal{L}_{5}^{j}+\mathcal{L}_{4}^{j}}{2} \\
d_{k}^{j} & =\mathcal{L}_{k}^{j}
\end{aligned}
$$

$$
\begin{aligned}
\mathcal{L}_{i=j}^{j} & =U_{j}\left(c^{2} \frac{\partial \rho}{\partial \breve{x}_{j}}-\frac{\partial P}{\partial \breve{x}_{j}}\right) \\
\mathcal{L}_{i \neq j}^{j} & =U_{j} \frac{\partial U_{i}}{\partial x_{j}} \\
\mathcal{L}_{4}^{j} & =\left(U_{j}-c\right)\left(\frac{\partial P}{\partial x_{j}}-\rho c \frac{\partial U_{j}}{\partial x_{j}}\right) \\
\mathcal{L}_{5}^{j} & =\left(U_{j}+c\right)\left(\frac{\partial P}{\partial x_{j}}+\rho c \frac{\partial U_{j}}{\partial \breve{x}_{j}}\right) \\
\mathcal{L}_{k}^{j} & =U_{j} \frac{\partial Y_{k}}{\partial x_{j}}
\end{aligned}
$$

When characteristics exit the computational domain, amplitudes $\mathcal{L}_{I}$ can be computed from inner points of the computational domain : their analytical expressions are given by system (4). On the other hand, characteristics entering the domain are to be modeled. For instance, at a subsonic outflow of normal vector $\vec{x}_{1}$, only acoustic wave amplitude $\mathcal{L}_{4}^{1}$ (cf equations $4 \mathrm{c}$ ) does bring information from outside (at speed $U_{1}-c$ ). Amplitude $\mathcal{L}_{4}^{1}$ has hence to be modeled. In [12], Poinsot \& Lele used a pressure relaxation term to prescribe this incoming acoustic wave. As previously mentioned, additional terms to this original formulation have been added to take into account transverse pressure and velocity variations $[13,14,27]$. In the present paper, for the sake of comparison, we shall make use of the same treatment as [13] to prescribe incoming characteristics for 3D-NSCBC subsonic outflows or subsonic inflows.

\subsection{Grid arrangements}

We now introduce the different grids adopted in the present strategy. Let consider a rectangular physical domain of length $L_{i}$ in the $i^{t h}$ direction (for $i=1,2$ or 3 ). This fluid domain is divided in 
rectangular cells, constituting a structured mesh. Fluid properties are assumed to be known at the nodes of this mesh. A collection of these nodes constitutes a grid. Physical variables can be arranged on different grid configurations (or arrangements), namely colocated, staggered or the present one, called hybrid. Figures $1 \mathrm{a}, 1 \mathrm{~b}$ and $1 \mathrm{c}$ respectively display these three kinds of arrangements for a line of length $L_{i}$ in the $i^{\text {th }}$ direction.

(a) colocated:

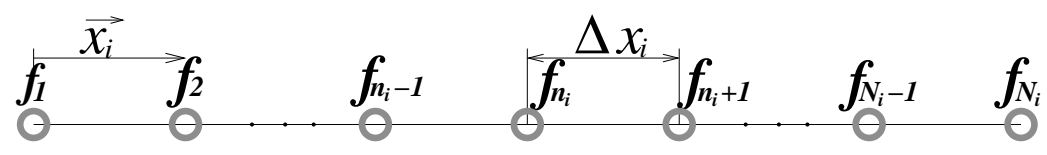

(b) Staggered :

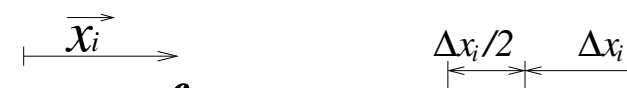

(c) Hybrid :

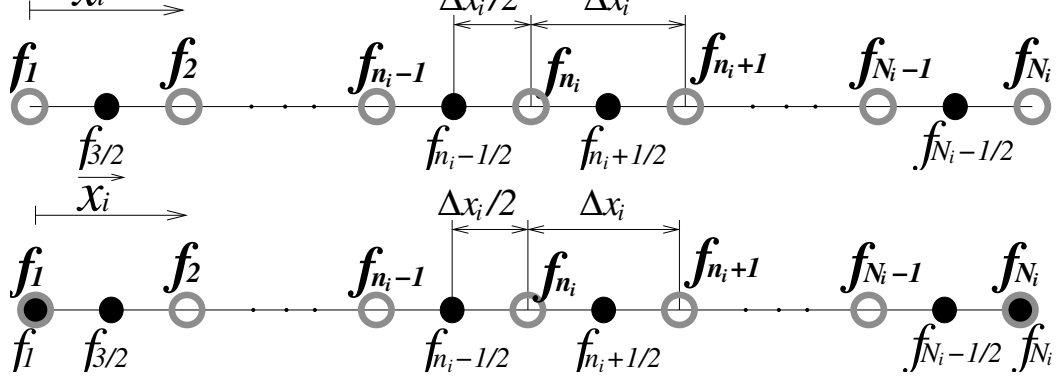

Figure 1: One-dimensional discretization on different grids. Full black dots : grid 'c', gray circles : grid 'f'. a: colocated (only one grid) ; b: staggered ; c: hybrid colocated-staggered.

With the colocated arrangement, a unique grid is used to discretize all physical variables (cf. figure 1a). With staggered or 'hybrid' strategies, more than one grid, staggered in space, are introduced. Scalar variables $\left(\rho, \rho E\right.$ or $\left.\rho Y_{k}\right)$ are stored on a cell-centered grid, grid 'c', while momentum (vector) components $\rho U_{i}$ are stored on a different face-centered grid 'f' (see figure $1 \mathrm{~b}$ and 1c). Hence, scalar values $f_{n_{i}+1 / 2}$ and vector components $f_{n_{i}}$ are meant to be evaluated on separate grids[2, 6, 9, 10]. Pressure $P$ and velocity components $U_{i}$ are shifted half a stepsize in the $i^{\text {th }}$ direction. For the present hybrid strategy, we add an extra half shifted point at the boundaries of grid 'c' (see figure 1c). This simple addition now allows scalars and vector components to be colocated at boundaries, while preserving the usual staggered arrangement within the fluid. As already mentioned, boundaries are unambiguously localized in the hybrid strategy. We lay the stress upon the fact that this is not the case in the staggered arrangements of [9, 10].

All grids can be straightforwardly deduced from a combination of lines 'grid c' and 'grid f' for 2D (cf. figure 2) or 3D arrangements (see table 1). In more than one dimension, these grids, combinations of elementary 1D grids 'c' and ' $f$ ', shall now be called grid $S$ (for 'scalar' grid) and grids $V_{i}$ (for 'vector' component grid). Grid $V_{i}$ is shifted half a stepsize in the $i$-th direction relatively to grid $S$ (cf. figure 2 and table 1). With the hybrid arrangement, no extrapolation is needed to express a scalar value on grid $V_{i}$. Notice that grids $V_{i}$ are uniform but grid $S$ is not; at the boundaries, points 1 and $3 / 2$ or points $N-1 / 2$ and $N$ of grid $S$ are separated by $\Delta x_{i} / 2$.

In addition to grids $S$ and $V_{i}$, other grids, denoted $T_{i j}$ with $i \neq j$, are introduced to minimize the number of required interpolations for the computations of some tensorial terms appearing in Navier-Stokes equations $\left(\tau_{i j}\right.$ or $\left.\rho U_{i} U_{j}\right)$. Relative to grid $V_{j}$, grid $T_{i j}$ is staggered in the $i^{\text {th }}$ direction (cf figure 2c and table 1). Grid $T_{i j}$ is then staggered in both $i^{\text {th }}$ and $j^{\text {th }}$ directions, with respect to scalar grid $S$, thus $T_{i j}$ and $T_{j i}$ grids are identical. Note that $T_{i j}$ coincide with the colocated grid 
(a) colocated

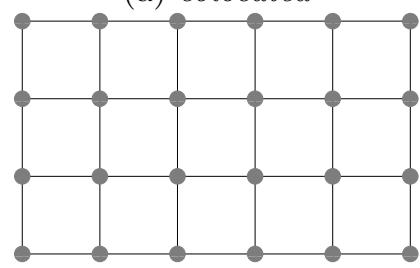

(b) Staggered

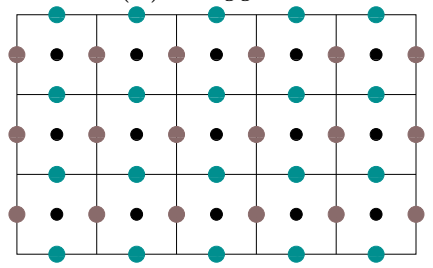

(c) Hybrid

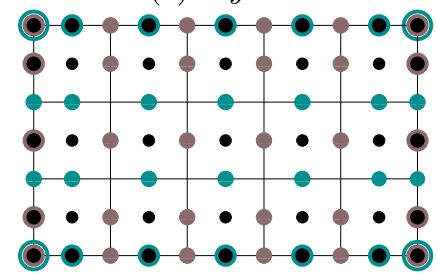

Figure 2: Two-dimensional discretization on different grids a: colocated (only one grid) ; b: staggered ; c: hybrid colocated-staggered. In the hybrid case, the various introduced grids are grid $S, \operatorname{grid} V_{i}$, grid $V_{j}$, and grid $T_{i j}$. Their elementary decomposition is given table 1 .

Table 1: Definition of different grids as a combination of elementary grid lines ' $\mathrm{f}$ ' and 'c' (see figure 1 ). Indexes $i, j$ and $k$ represent directions of grids $S, V_{i}$ and $T_{i j}$. Integers $N P_{\text {stag }}$ and $N P_{h y b}$ represent respectively the number of points in staggered and hybrid grids. This table also describes $2 \mathrm{D}$ grids of figure 2 if the $k^{\text {th }}$ direction is forgotten.

\begin{tabular}{c||c|c|c|r|r}
\hline Grids & $i$ & $j$ & $k$ & \multicolumn{1}{c}{$N P_{\text {stag }}$} & \multicolumn{1}{c}{$N P_{h y b}$} \\
\hline \hline$S$ & $\mathrm{c}$ & $\mathrm{c}$ & $\mathrm{c}$ & $\left(N_{i}-1\right)\left(N_{j}-1\right)\left(N_{k}-1\right)$ & $\left(N_{i}+1\right)\left(N_{j}+1\right)\left(N_{k}+1\right)$ \\
\hline$V_{i}$ & $\mathrm{f}$ & $\mathrm{c}$ & $\mathrm{c}$ & $N_{i}\left(N_{j}-1\right)\left(N_{k}-1\right)$ & $N_{i}\left(N_{j}+1\right)\left(N_{k}+1\right)$ \\
\hline$T_{i j}$ & $\mathrm{f}$ & $\mathrm{f}$ & $\mathrm{c}$ & $N_{i} N_{j}\left(N_{k}-1\right)$ & $N_{i} N_{j}\left(N_{k}+1\right)$ \\
\hline
\end{tabular}

in 2D (cf. figure 2) but does not coincide with the colocated grid in 3D (cf. table 1) since they are staggered in the $k$-th direction.

In $3 \mathrm{D}$, this hybrid approach a priori requires 7 different grids (grid $S, 3$ grids $V_{i}$ and 3 grids $T_{i j}$ ) while only one grid is needed for a colocated arrangement. But actual data storage increase is minimal. Instead of storing variables in a $N_{1} \times N_{2} \times N_{3}$ array on a single colocated grid, variables are conveniently stored in a $\left(N_{1}+1\right) \times\left(N_{2}+1\right) \times\left(N_{3}+1\right)$ array. Since usually $N_{i} \geqslant 100$, effective data storage increase is less than a few percent (less than $0.3 \%$ when $N_{i} \simeq 1000$ ).

\subsection{Differentiation and interpolation operators.}

Despite apparent complexity, only two different schemes of first derivative and interpolation are needed to differentiate a field from one grid to another. We note 'Interp1' the scheme allowing to interpolate from grid ' $c$ ' to grid ' $f$ ' and 'Interp2' the scheme allowing to interpolate discrete values from grid 'f' to grid 'c'. Similarly, operator 'Diff1' differentiates variables from grid 'c' to grid 'f' whereas operator 'Diff2' does the same from grid ' $f$ ' to grid 'c'.

In $1 \mathrm{D}$, the 4 different introduced operators are then :

- Interp1 $1_{c \rightarrow f} \quad$ Diff1 $1_{c \rightarrow f}$

- Interp2 $2_{f \rightarrow c} \quad$ Diff2 $2_{f \rightarrow c}$

Since multidimensional arrangements are combinations of 1D arrangements, the same operators can be used to differentiate or interpolate in 3D. For instance, to differentiate from grid $S=$ 'ccc' to grid $V_{1}=$ 'fcc' in direction $\overrightarrow{x_{1}}$, operator Diff1 is used to compute derivatives from each line 'c' to each line ' $\mathrm{f}$ '. This operation is carried out for all lines along $\overrightarrow{x_{1}}$ direction. For multidimensional 
problems, possible different operations of differentiation and interpolation are :

- Interp $1_{S \rightarrow V_{i}} \quad \operatorname{Diff}_{S \rightarrow V_{i}}$

- Interp1 $1_{V_{i} \rightarrow T_{i j}} \quad$ Diff1 $1_{V_{i} \rightarrow T_{i j}}$

- Interp2 $2_{V_{i} \rightarrow S} \quad$ Diff $2_{V_{i} \rightarrow S}$

- Interp2 $2_{T_{i j} \rightarrow V_{i}} \quad \operatorname{Diff} 2_{T_{i j} \rightarrow V_{i}}$

The hybrid approach seems to be essentially a staggered approach with an extra point at the boundary. However, how this will be analysed next section, the behaviour of finite difference schemes substantially changes, both in terms of accuracy and stability. Moreover, boundary condition treatment - in particular for characteristic boundary conditions, like NSCBC treatment [12] or 3DNSCBC $[13,14]$ - is now simple and natural, like in the pure colocated arrangement.

As in the present strategy, Nagarajan et al. [9] make use of two derivative schemes and two interpolation schemes to solve Navier-Stokes equations. These schemes allow to differentiate from one grid to another [10]. Next section, we shall compare their finite difference schemes with the present one.

\section{ANALYSIS OF FINITE DIFFERENCE SCHEMES}

In this section, we briefly introduce the notations used for finite difference schemes. We then lead a (classical) Fourier analysis of the three considered schemes, namely the colocated, staggered and hybrid schemes.

\subsection{Finite difference schemes}

Let $f$ be a sufficiently smooth function, defined from $[0, L] \subset \mathbb{R}$ to $\mathbb{C}$ and let $\Delta x=L /(M-1)$ denote a given spatial step size; $M$ is the number of grid points, and $n$ is an integer or half-integer varying from 0 to $M-1$. To numerically interpolate or differentiate the function $f(x)$ at point $x_{n}=n \Delta x$ with an order $N$ formal precision, linear relations respectively of the form (5a) and (5b) can be introduced:

$$
\begin{aligned}
\bar{\alpha}_{0} \bar{f}_{n}+\sum_{k=1}^{N_{\alpha}} \bar{\alpha}_{k} \bar{f}_{n+a_{k}} & =\sum_{k=1}^{N_{\beta}} \bar{\beta}_{k} f_{n+b_{k}}+\mathcal{O}\left(|\Delta x|^{N}\right) \\
\dot{\alpha}_{0} \dot{f}_{n}+\sum_{k=1}^{N_{\alpha}} \dot{\alpha}_{k} \dot{f}_{n+a_{k}} & =\frac{1}{\Delta x} \sum_{k=1}^{N_{\beta}+1} \dot{\beta}_{k} f_{n+b_{k}}+\mathcal{O}\left(|\Delta x|^{N}\right)
\end{aligned}
$$

where $f_{n+a_{k}}=f\left(x(n)+a_{k} \Delta x\right), f_{n+b_{k}}=f\left(x(n)+b_{k} \Delta x\right) ; a_{k}$ and $b_{k}$ are real numbers (here half integers); $N_{\alpha}$ and $N_{\beta}$ are integers such that $N_{\alpha}+N_{\beta}=N$. The $\left(N_{\alpha}+N_{\beta}\right)$ coefficients $\left(a_{1}, \ldots, a_{N_{\alpha}}, b_{1}, \ldots, b_{N_{\beta}}\right)$ define the stencil of the interpolation or the differentiation formula. Weighting coefficients $\left(\bar{\alpha}_{k}, \bar{\beta}_{k}, \dot{\alpha}_{k}, \dot{\beta}_{k}\right)$ depend on the adopted stencil. The way they may be computed, in particular for present hybrid arrangement, is discussed in the appendix. The appendix also details the used finite difference schemes at boundary or inner points for colocated, staggered or hybrid arrangements. As soon as $N_{\alpha} \geqslant 1$, the scheme becomes implicit (so called Padé schemes [6]) and requires inversion of a linear system. In the following, only tridiagonal systems $\left(N_{\alpha}=2\right)$ will be considered. These systems can be conveniently inverted by Thomas algorithm, which only requires $\mathcal{O}(M)$ operations [15]. 
Since present work is mainly devoted to boundary treatment influence on global accuracy, only $6^{\text {th }}$ order schemes (for inner points) will be considered here (these schemes offer a good compromise between computational cost and accuracy [6,3]). Both implicit and explicit $6^{\text {th }}$ order schemes are presented. At the boundaries, one-sided stencils are used, implying a formal decrease of the order of accuracy. Orders are decreased to keep a constant stencil size throughout the computational domain since instabilities often occur when stencil width is increased $[17,18]$. With explicit schemes, three points are used on each side of the current point. The size of the stencil is then $3 \Delta x$ and $2.5 \Delta x$ for respectively the colocated arrangement or the staggered arrangement. With implicit schemes, stencils are more compact : their sizes are respectively $2 \Delta x$ and $1.5 \Delta x$.

\section{$2.2 \quad$ Fourier analysis}

We now apply Fourier analysis of errors to 1D differentiation and interpolation schemes and introduce transfer functions. We shall be able to compare the accuracy of these finite-difference schemes for these three kind of arrangements. In this subsection, we denote with an asterisk ${ }^{*}$ dimensional values: $\Delta x^{*}=L /(M-1)$ and $x^{*}=n \Delta x^{*}$. As in [6, 1, 21], we define a normalized wave number $\omega=\omega^{*} \Delta x^{*}(\omega \in[0, \pi])$ and a normalized coordinate $x=x^{*} / \Delta x^{*}(x=n \Delta x \in[0, M-1]$, with $\Delta x=1)$ such that $\omega^{*} x^{*}=\omega x$.

For the differentiation scheme, substituting $\dot{f}_{n+a_{k}}$ by $i \omega e^{i \omega\left(n+a_{k}\right)}$ and $f_{n+b_{k}}$ by $e^{i \omega\left(n+b_{k}\right)}$, equation $(5 \mathrm{~b})$ yields :

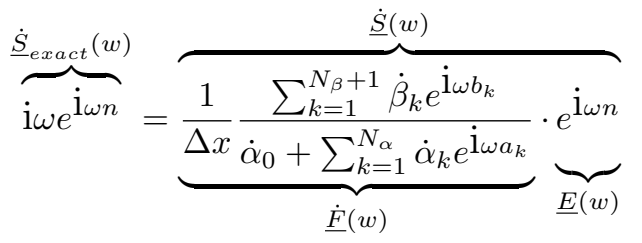

Equation (6) indicates that the response $\underline{\dot{S}}(w)$ of a particular scheme (5b) to an elementary trigonometric input $\underline{E}(w)=e^{i \omega x}$ can be characterized by a transfer function $\underline{\dot{F}}(w)$. The difference between the transfer function $\underline{\underline{F}}(w)$ and its exact (spectral) value $i \omega$ for any $\omega \in[0 ; \pi[$ qualifies the accuracy of the considered FD scheme. The difference between the modulus $\|\dot{F}\|$ and $\omega$ represents the dispersive error of the scheme while the difference between the argument $\arg (\underline{\bar{F}})$ and the exact value $\pi / 2$ is the dissipative error. When stencil is symmetrical, transfer function of differential scheme becomes purely imaginary [6] and the finite difference scheme has no dissipative error [20].

Interpolation schemes of type (5a) can be analyzed in a similar way. The transfer function writes this time

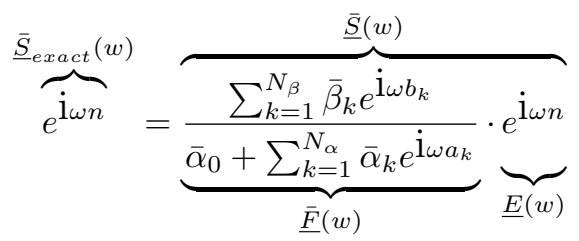

No interpolation occurs if transfer function $\underline{\bar{F}}$ is equal to one. Like in the derivative case, the difference between modulus $\|\underline{\bar{F}}\|$ and unity represents dispersive error of the interpolation FD scheme; the difference between $\arg (\underline{\bar{F}})$ and zero represents the dissipative error. For centered stencils, the transfer function is purely real and this FD scheme has no dissipative error. 
Figure 3 compares transfer function modulus of first-derivative schemes as a function of $\omega$ for $6^{\text {th }}$ order centered schemes. Explicit and implicit schemes for staggered and colocated arrangements are compared. The values of the coefficients are given Tables 5-6-7 in the Appendix. For inner points, no difference exists between hybrid and staggered schemes, while we shall see this is no more true close to the boundaries.

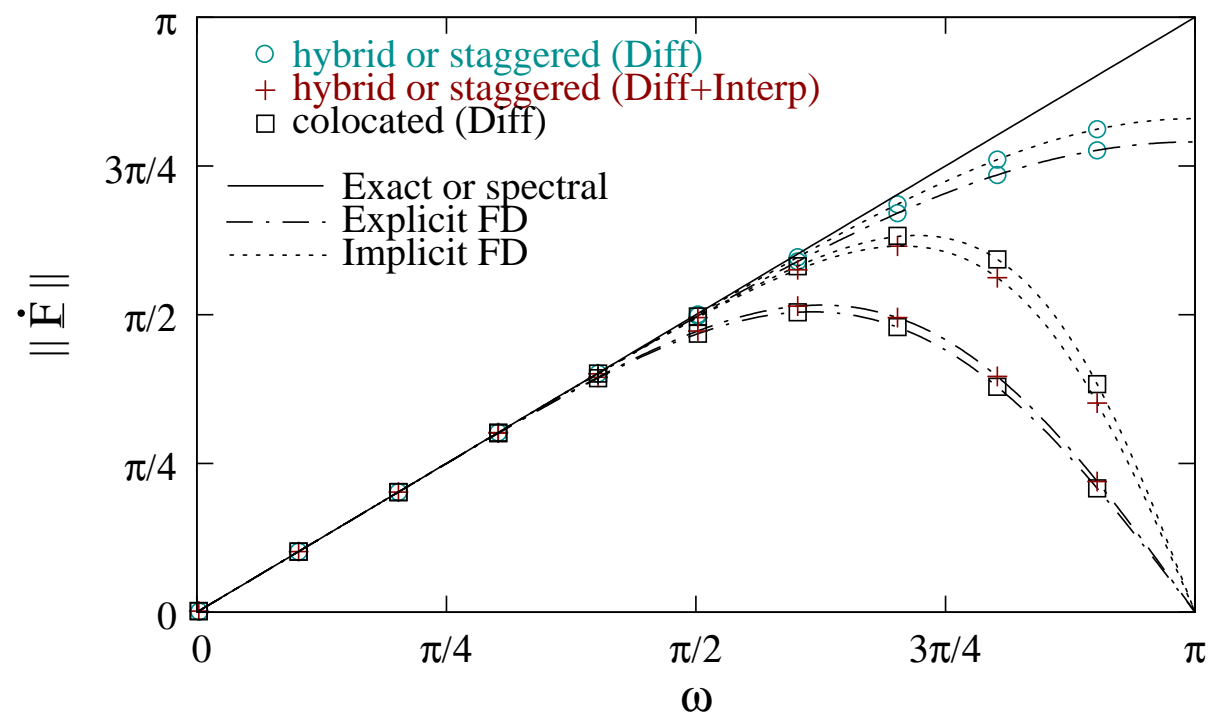

Figure 3: Comparison of centered 6-th order explicit and implicit (Padé) schemes. The modulus of transfer functions (see formulas C_6e, C_6i, Sd1_6e, Sd1_6i, Si1_6e, Si1_6i of the appendix) for hybrid, staggered and colocated arrangements are plotted.

As noticed in the literature [6,10], implicit (Padé) schemes are more accurate than explicit schemes. This means that for compact schemes less points are needed to obtain the same accuracy. It is worth noticing however, as shown figure 3, that (for the present analyzed schemes) this difference in accuracy is quite small, in particular for staggered schemes. Explicit staggered schemes seem good candidates for a practical implementation : for comparable accuracy (with respect to compact schemes), their CPU cost is cheaper and their parallel version is much easier to implement.

For staggered approaches, solving Navier-Stokes equations may require to successively interpolate and differentiate (see tables 2, 3,4). For centered schemes, when successively performing differentiation and interpolation operations - for instance to differentiate a quantity from a grid $G_{1}$ to $G_{1}, G_{1}$ being any of the previously introduced $S, V_{i}$ or $T_{i j}$ - it is simple to show that the equivalent transfer function is the product of the two transfer functions of the differentiation and interpolation operators :

$$
\underline{\dot{F}}_{G_{1} \rightarrow G_{1}}=\underline{\dot{F}}_{G_{1} \rightarrow G_{2}} \cdot \underline{\bar{F}}_{G_{2} \rightarrow G_{1}},
$$

$G_{1}$ and $G_{2}$ being two shifted grids. Figure 3 plots tranfer function modulus of the (Interp+Diff) operator for staggered grids. The corresponding curves indicate that overall accuracy of the (Interp+Diff) operator is almost the same as the one observed with a colocated scheme. To our knowledge, this has never been stressed on the literature. When numerically solving Navier-Stokes equations with a staggered strategy, some terms - computed with the (Interp+Diff) operator - 
will be evaluated with an accuracy comparable to a colocated scheme while others - computed with the Diff operator - will be evaluated with an improved accuracy. This means than this staggered scheme introduces less overall numerical error than the colocated one.

Figure 4 shows modulus and argument of transfer function for one-sided three-point explicit schemes used at boundaries. Schemes for differentiation (fig. $4 \mathrm{a}$ and $4 \mathrm{~b}$ ) and interpolation (fig. 4c and 4d) are compared for colocated, staggered or hybrid arrangements. Modulus and argument are respectively plotted figures $4 \mathrm{a}$ and $4 \mathrm{c}$ and figures $4 \mathrm{~b}$ and $4 \mathrm{~d}$. Figure 5 shows the same comparisons but for one-sided two-point implicit schemes.

(a)

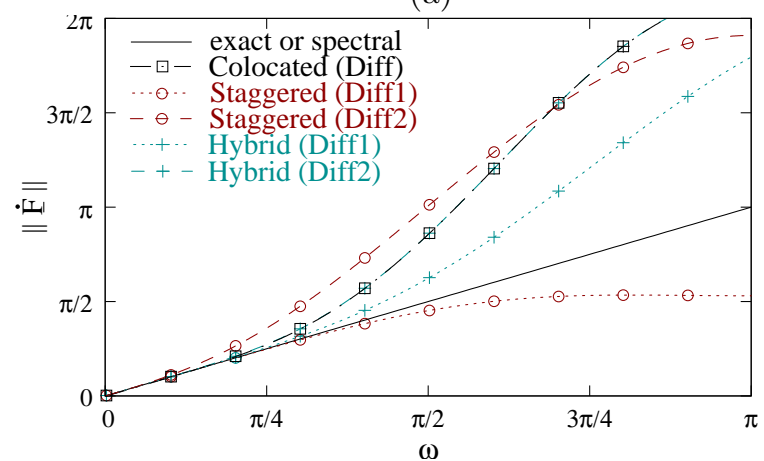

(c)

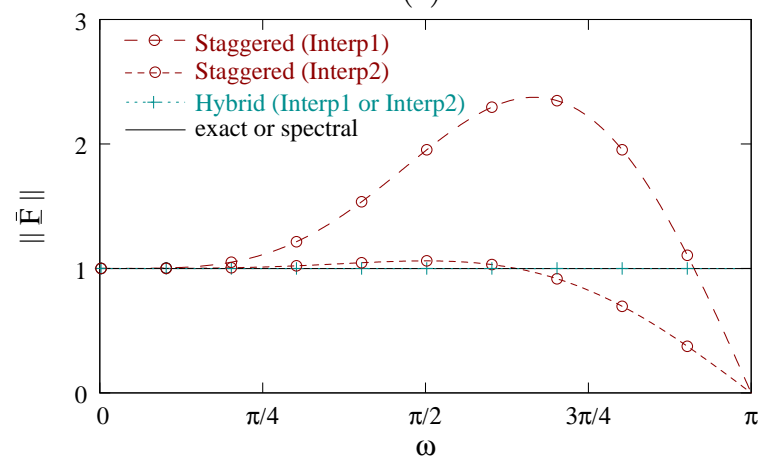

(b)

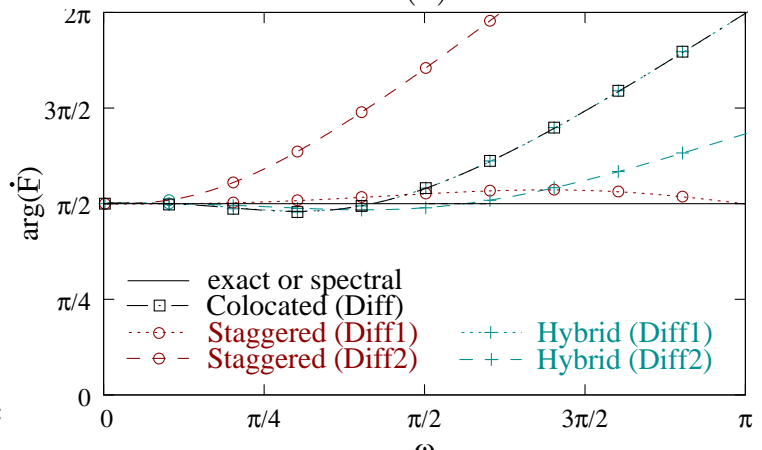

(d)

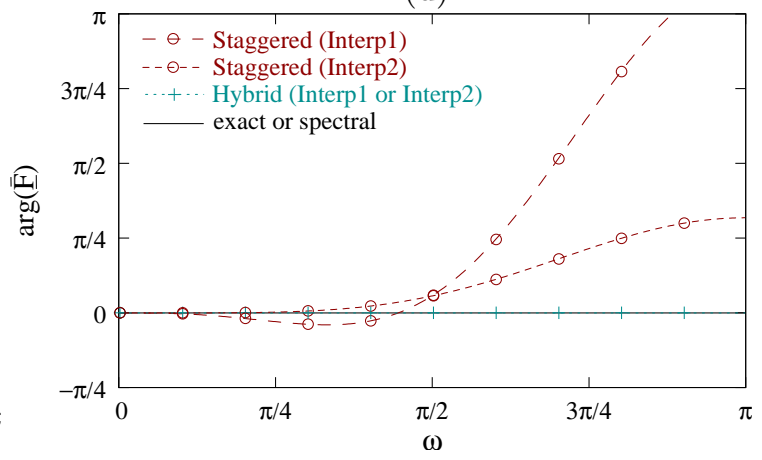

Figure 4: Comparison of one-sided three-point explicit finite difference schemes at boundary points. Modulus and argument of transfer function as a function of $\omega$, for colocated, staggered and hybrid schemes. (a,b) : formulas C_3e, Sd1_2e, Sd2_3e, Hd1_3e, Hd2_3e of the appendix. (c,d) : formulas Si1_3e, Si2_4e, Hi1_0e, Hi2_0e. Notice that Diff (Colocated) and Diff2 (Hybrid) are the same for (a) and (b).

In figures $4 \mathrm{a}$ and $5 \mathrm{a}$, modulus of transfer functions shows that hybrid arrangement of variables decreases the dispersive error at boundaries for first derivatives, with respect to colocated and staggered arrangements. This is particularly true for explicit schemes.

Figures $4 \mathrm{c}, 4 \mathrm{~d}, 5 \mathrm{c}$ or $5 \mathrm{~d}$ show that interpolation also generates errors at the boundaries in the staggered approach. With the colocated or hybrid arrangements, no interpolation occurs and error vanishes. 
(a)

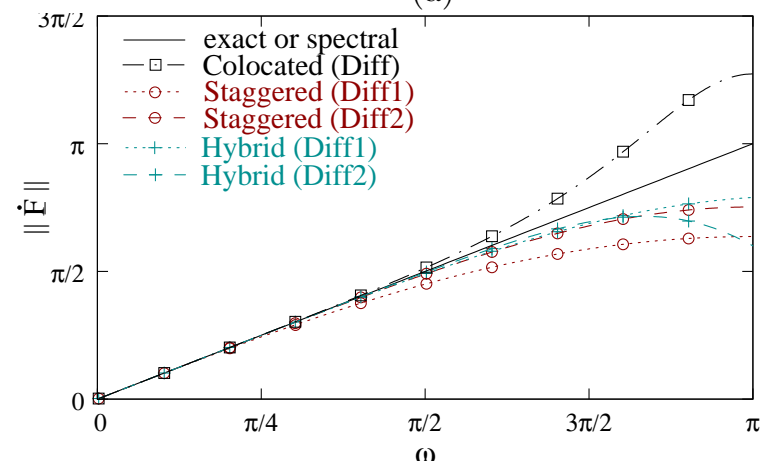

(c)

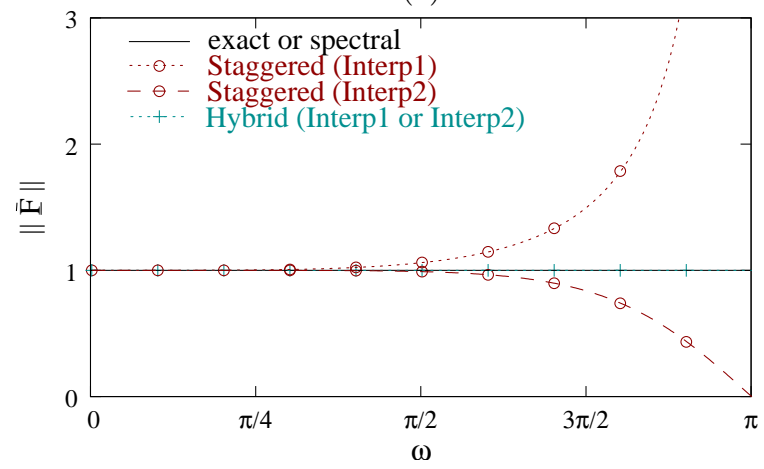

(b)

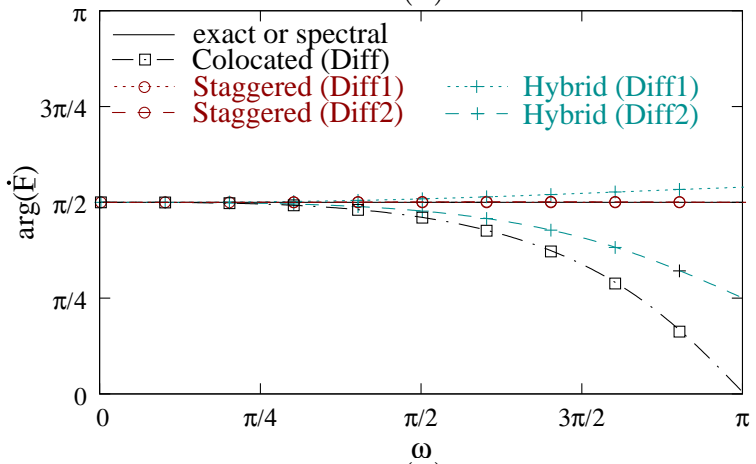

(d)

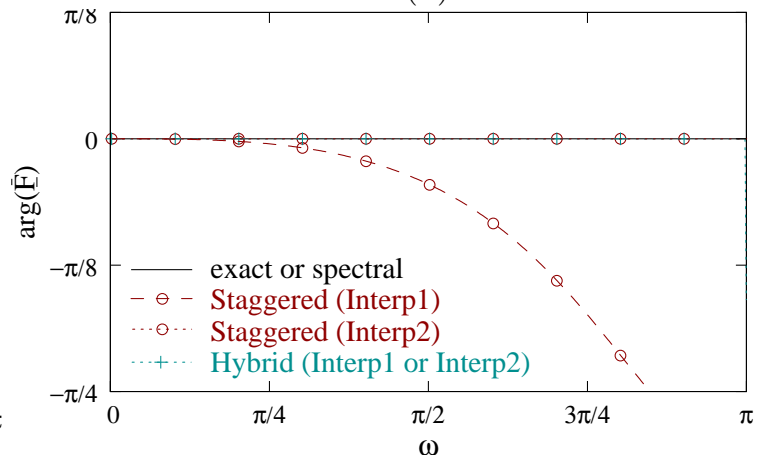

Figure 5: Comparison of one-sided two-point implicit finite difference schemes at boundary points. Modulus and argument of transfer function as a function of $\omega$, for colocated, staggered and hybrid schemes. (a,b) : formulas C__3i, Sd1_3i, Sd2_3i, Hd1_3i, Hd2_3i of the appendix. (c,d) : formulas Si1_3i, Si2_4i, Hi1_0i, Hi2_0i.

\section{APPLICATION TO NAVIER-STOKES EQUATIONS}

For the sake of completeness, we now describe how to apply the above differentiation and interpolations rules to the numerical solution of fully compressible reactive flow balance equations.

At the beginning of any one time-step, the $\rho, \rho E$ and $\rho Y_{k}$ scalar conservative variables are known on grid $S$ while the momentum components $\rho U_{i}$ are known on grid $V_{i}$ for $i=1,2,3$. If a variable $X$ is known at grid $G$, this will be denoted $\left.X\right|_{G}$.

In a first step, pressure, temperature, viscosity are computed on grid $S$, while velocity components are determined on grids $V_{i}$. Pressure and velocity gradients are also computed. This preliminary computational step is summarized table 2 .

Then, computation of momentum divergence is required (for continuity equation). Since density is a scalar, its time derivative $\partial \rho / \partial t$ is to be estimated on the cell-centered grid $S$. The momentum components $\rho U_{i}$ are stored on the face-centered grids $V_{i}$. Using operator Diff2 $V_{i} \rightarrow S$, momentum divergence computation on grid $S$ is straightforward and does not require any extra interpolation. Tables 3 and 4 respectively summarize how mass and momentum time-evolutions are computed. Energy and scalar computations are similar (they are not included for the sake of brevity). To 
Table 2: Preliminary steps for Navier-Stokes discretization.

\begin{tabular}{|c|c|c|}
\hline Used Variable & Operator & Result: needed quantities \\
\hline$\left.\rho\right|_{S}$ & Interp $_{S \rightarrow V_{i}}$ & $\left.\rho\right|_{V_{i}}$ \\
\hline$\left.\rho\right|_{V_{i}},\left.\rho U_{i}\right|_{V_{i}}$ & Divide & $\left.U_{i}\right|_{V_{i}}=\left.\rho U_{i}\right|_{V_{i}} /\left.\rho\right|_{V_{i}}$ \\
\hline$\left.U_{i}\right|_{V_{i}}$ & Diff2 $V_{i} \rightarrow S$ & $\partial U_{i} /\left.\partial x_{i}\right|_{S}$ \\
\hline$\left.U_{i}\right|_{V_{i}}$ & $\operatorname{Diff}_{V_{i} \rightarrow T_{i j}} \quad(j \neq i)$ & $\partial U_{i} /\left.\partial x_{j}\right|_{T_{i j}}$ \\
\hline$\left.\rho U_{i}\right|_{V_{i}}$ & Interp $2_{V_{i} \rightarrow S}$ & $\left.\rho U_{i}\right|_{S}$ \\
\hline$\rho,\left.\rho U_{i}\right|_{S}$ & Simple Algebra & $\left.\rho U_{k}^{2}\right|_{S}$ \\
\hline \multirow[t]{2}{*}{$\rho,\left.\rho E\right|_{S}$} & Simple Algebra & $\left.T\right|_{S} \equiv\left(\rho E-\rho U_{k}^{2} / 2\right) /\left.\rho C_{V}\right|_{S}$ \\
\hline & Simple Algebra & $\left.P\right|_{S}=\left.\rho r T\right|_{S}$ \\
\hline$\left.P\right|_{S}$ & $\operatorname{Diff}_{S \rightarrow V_{i}}$ & $\partial P /\left.\partial x_{i}\right|_{V_{i}}$ \\
\hline
\end{tabular}

Table 3: Calculation of the continuity equation RHS.

\begin{tabular}{lll}
\hline Used Variable & Operator & Result: needed quantities \\
\hline \hline$\left.\rho U_{j}\right|_{V_{j}}$ & ${\text { Diff }{ }_{V_{j} \rightarrow S} / j}$ & $\partial \rho U_{j} /\left.\partial x_{j}\right|_{S}$ \\
\hline
\end{tabular}

march in time, we adopt the minimal-storage strategy described in [28]. The algorithm consists in a third order Runge-Kutta integration scheme that only requires two memory locations for each conserved property.

For boundary NSCBC treatment (see systems (3), (4) ) all wave amplitudes $\mathcal{L}_{i}$ and convective terms $d_{i}$ are computed on scalar grid $S$. As is usual with "improved NSCBC" [13, 14], all needed terms are stored at the boundary face points of grid $S$, and, when needed, interpolations are carried out only at boundaries. The only difference with the colocated method is that momentum time derivatives estimated by NSCBC theory on grid $S$ have to be interpolated on grid $V_{i}$. Time derivative term $\left\{\partial \rho U_{i} / \partial t\right\}_{N S C B C}$ also has to be interpolated with the Interp $1_{S \rightarrow V_{i}}$ on faces colinear to the $i^{\text {th }}$ direction.

Next sections, the present methodology will now be applied on 1D non reactive and reactive tests and also on 2D and 3D flame computations.

\section{OUTGOING PRESSURE WAVE TEST}

In this section, the non-reflecting outlet of the hybrid arrangement is tested and compared to both staggered and colocated arrangements. As in [9], we simulate a one-dimensional acoustic wave with non-reflecting left and right boundaries. Standard NSCBC treatment with present hybrid scheme is used to allow direct comparison with the staggered NSCBC treatment of [9].

Velocity profile is initialized as a Gaussian in the middle of a unit length 1D domain. This initial condition generates two travelling acoustic waves propagating to left and right boundaries. Since these waves propagate at Mach number $\mathrm{Ma}=1$, they should reach the boundaries for the first time at acoustic time $t=0.5$. Numerically, these waves are partially reflected at the boundaries and the reflected waves will propagate back, interact, exit to be partially reflected again on the opposite side at time $t=1.5$. The process is likely to proceed until waves amplitude vanishes. Time evolution of pressure fluctuation rms is an indicator of BC treatment influence [9].

Figure $6 \mathrm{a}$ presents the pressure rms time evolution for initial Gaussian pulse discretized with 
Table 4: Computation of $i^{\text {th }}$ momentum equation RHS.

\begin{tabular}{|c|c|c|}
\hline Used Variable & Operator & Result: needed quantities \\
\hline \multirow[t]{3}{*}{$\rho U_{i},\left.U_{i}\right|_{V_{i}}$} & Multiply & $\left.\rho U_{i} U_{i}\right|_{V_{i}}$ \\
\hline & Diff2 $V_{V_{i} \rightarrow S}$ & $\partial \rho U_{i} U_{i} /\left.\partial x_{i}\right|_{S}$ \\
\hline & Interp $_{S \rightarrow V_{i}}$ & $\partial \rho U_{i} U_{i} /\left.\partial x_{i}\right|_{V_{i}}$ \\
\hline \multirow{4}{*}{$\begin{array}{l}\left.\rho U_{j}\right|_{V_{j}} \\
\left.U_{i}\right|_{V_{i}}\end{array}$} & Interp1 $_{V_{j} \rightarrow T_{j i}} \quad(i \neq j)$ & $\left.\rho U_{j}\right|_{T_{j i}}$ \\
\hline & $\operatorname{Interp}_{V_{i} \rightarrow T_{i j}} \quad(j \neq i)$ & $\left.U_{i}\right|_{T_{i j}}$ \\
\hline & Multiply & $\left.\rho U_{i} U_{j}\right|_{T_{i j}}$ \\
\hline & $\operatorname{Diff}_{T_{i j} \rightarrow V_{i}} \quad(j \neq i)$ & $\partial \rho U_{i} U_{j} /\left.\partial x_{j}\right|_{V_{i}}$ \\
\hline$\left.P\right|_{S}$ & $\operatorname{Diff}_{S \rightarrow V_{i}}$ & $\overline{\partial P /\left.\partial x_{i}\right|_{V_{i}}}$ \\
\hline$\overline{\mu, \partial U_{i} /\left.\partial x_{i}\right|_{S}}$ & $\begin{array}{l}\text { Simple algebra } \\
\text { Diff1 } 1_{S \rightarrow V_{i}}\end{array}$ & $\begin{array}{l}\mu\left(2 \partial U_{i} / \partial x_{i}-2 / 3 \cdot \operatorname{div} \boldsymbol{U}\right)=\left.\tau_{i i}\right|_{S} \\
\partial \tau_{i i} /\left.\partial x_{i}\right|_{V_{i}}\end{array}$ \\
\hline $\begin{array}{l}\left.\mu\right|_{S} \\
\left.\mu\right|_{V_{i}} \\
\partial U_{i} /\left.\partial x_{j}\right|_{T_{i j}}\end{array}$ & $\begin{array}{l}\text { Interp1 }_{S \rightarrow V_{i}} \\
\text { Interp1 }_{V_{i} \rightarrow T_{i j}} \quad(i \neq j) \\
\text { Multiply } \\
\text { Diff2 }_{T_{i j} \rightarrow V_{i}} \quad(j \neq i)\end{array}$ & $\begin{array}{l}\left.\mu\right|_{V_{i}} \\
\left.\mu\right|_{T_{i j}} \\
\mu\left(\partial U_{i} / \partial x_{j}+\partial U_{j} / \partial x_{i}\right)=\left.\tau_{i j}\right|_{T_{i j}} \\
\partial \tau_{i j} /\left.\partial x_{j}\right|_{V_{i}}\end{array}$ \\
\hline
\end{tabular}

4 points, while fig. $6 \mathrm{~b}$ plots the same for a 16 -point Gaussian pulse. The reflection coefficient is defined as the ratio of the rms before and after the wave exits the domain. In the resolved 16-point case, this coefficient varies from $1 \%$ (staggered case of [9]) to $0.4 \%$ (colocated) and $0.1 \%$ (hybrid). In the poorly resolved 4 -point case, the values are respectively close to $40 \%, 25 \%$ and $10 \%$. In both cases, significant improvement of pulse absorption is observed for the present hybrid arrangement.

Moreover, when only four points are used to define the Gaussian pressure fluctuation, the colocated arrangement does not have enough points to correctly convect the acoustic wave. Oscillations appear before the fluctuation actually reaches the boundaries. In the staggered case, some unphysical increase of the rms appears when the wave pulse reaches the boundary. This is apparently due to boundary point treatment, where extrapolations are needed in this "pure" staggered approach. On the contrary, behaviour for the hybrid arrangement remains pertinent, even for this poorly refined case.

\section{PREMIXED FLAME TESTS}

\subsection{Laminar 1D premixed flame}

We also performed one-dimensional premixed stoichiometric methane-air flame computation. The hybrid simulation is compared with the colocated simulation. We remind that, for simplicity, a single step Arrhenius law for chemistry is assumed. Fresh gas is injected with a 'soft-inflow' [13]. The use of a 'hard inflow' [12] has been checked not to change next observations. The flame propagates to the inflow while hot gases are expanding through an 'improved non-reflecting outlet' (cf. [13]). Figure 7 a presents the temperature, pressure and reaction rate profiles for this propagating laminar flame. Mesh refinement is varied in order to investigate the behaviour of the flame simulation when less mesh points are used. The simulations are carried out with both colocated and hybrid arrangements and with two different numerical schemes : an explicit high order finite difference scheme (FD) and an implicit high order compact scheme (Padé) as described section 2. Figure 7b plots normalized 
(a)

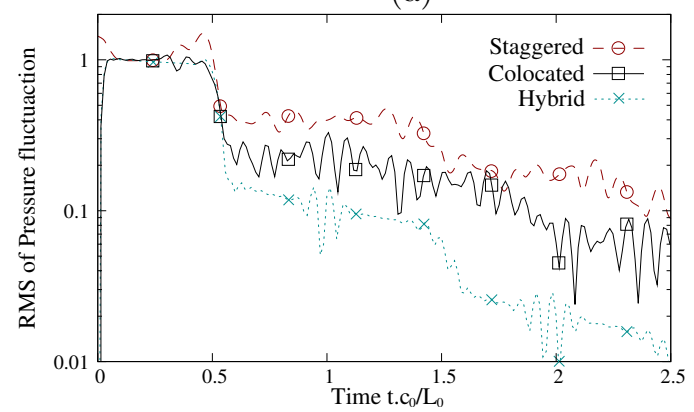

(b)

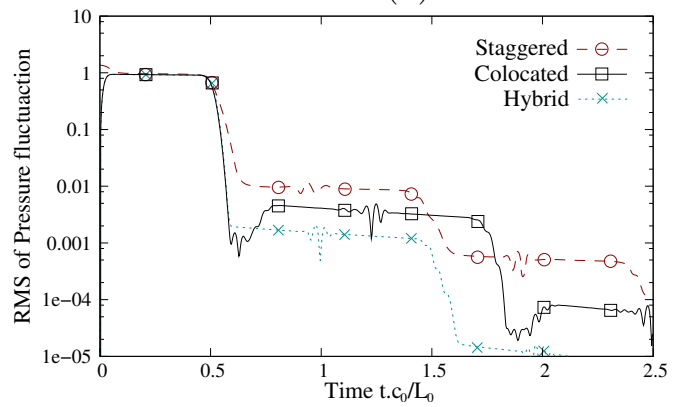

Figure 6: Simulation of outgoing acoustic waves generated by an initial Gaussian velocity pulse: rms of the pressure fluctuation time evolution with respectively 4 points (a) or 16 points (b) for discretizing the initial Gaussian. circles: staggered calculation of [9], crosses: hybrid (present strategy), squares: colocated method.

CPU time vs mesh resolution. For a given resolution, hybrid scheme is more expensive than the colocated one, since it requires extra interpolations. However, as will be emphasized below, similar accuracy can be obtained with approximately twice less refined meshes for the hybrid approach. Moreover, when resolution is too low, colocated scheme leads to numerical instability (negative temperature). This is indicated by the missing points (squares) of figure at low resolution. For a given accuracy, and as expected, hybrid explicit schemes are faster than implicit (that requires to invert a tridiagonal system).

(a)

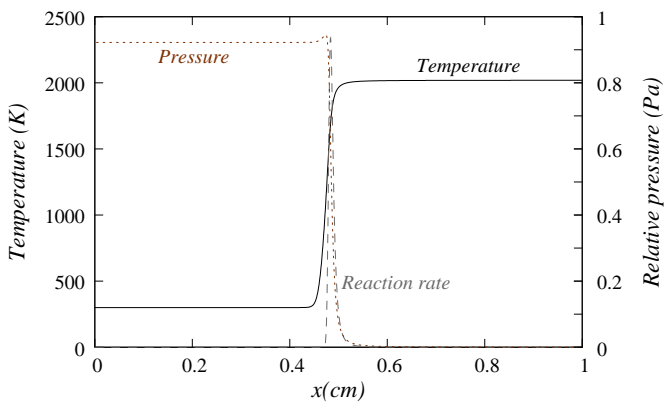

(b)

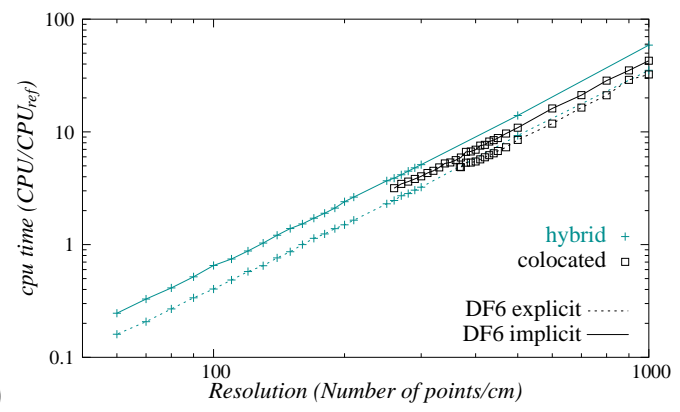

Figure 7: 1D laminar flame simulation. (a) profiles of temperature, pressure and reaction rate. (b) cpu-time along the resolution for different numerical strategy : hybrid or colocated arrangements, explicit or implicit $6^{\text {th }}$ order schemes.

Figure 8a presents computed laminar flame velocity as a function of the number of points in the reaction zone (of $\delta_{r}$ thickness) while figure $8 \mathrm{~b}$ plots the computed pressure jump.

For sufficiently refined solutions, all computations yield similar results with a laminar flame speed $S_{L} \simeq 0.38 \mathrm{~m} / \mathrm{s}$ and a pressure jump $\Delta P=\rho_{u} S_{L}^{2} \alpha /(1-\alpha) \simeq 0.9 P a$. In figure 8a, if the number of grid points used to discretize $\delta_{r}$ is below 20 (resp. 26) for the implicit colocated method (resp. explicit colocated), the computed flame velocity increases and the simulation quickly leads to strong oscillations and unphysical behaviour. In particular, unphysical pressure drops appears 


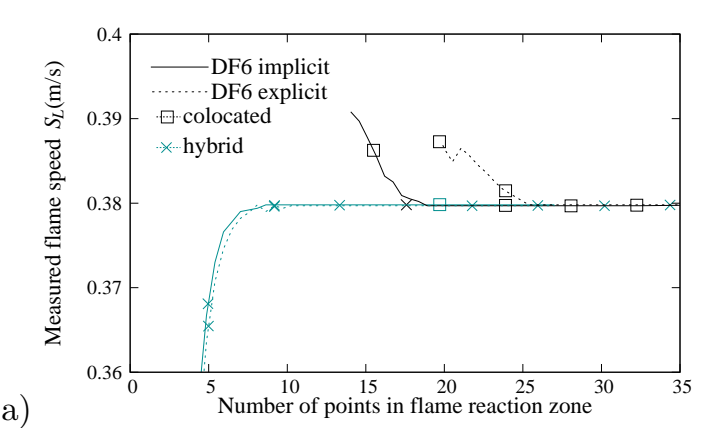

(a)

(b)

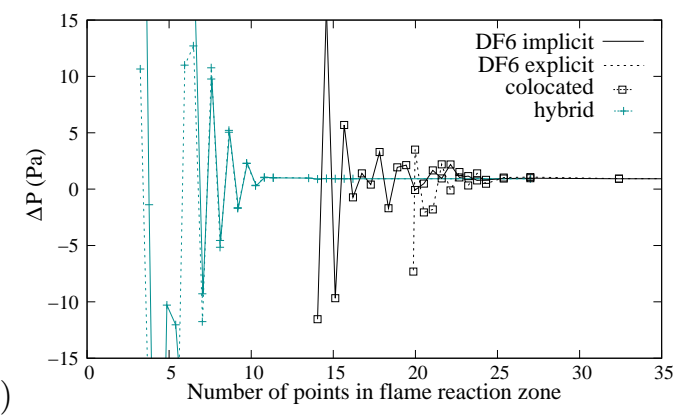

Figure 8: Measured laminar flame speed vs reaction zone refinement. Different numerical schemes and grid arrangements are used. Dotted lines: $6^{\text {th }}$ order finite difference scheme, full lines: $6^{\text {th }}$ order Padé scheme ; squares: colocated arrangement, crosses: hybrid arrangement.

between boundary points and domain inner points at the outlet and at the inlet. On the contrary, for the present strategy, the computed laminar flame velocity still remains correct down to 10point resolution and no boundary effects are observed. Figure $8 \mathrm{~b}$ also indicates that the hybrid arrangement allows for a correct evaluation of pressure jump, with a lower resolution than with colocated simulations.

These results suggest that the observed instability for the Padé and the FD comes from unsufficient resolution of the flame thickness and of boundary conditions. With low resolutions, the hybrid arrangement is shown to lead to more pertinent results than with a colocated arrangement of variables. No boundary effects are observed and correct laminar flame speed and pressure drops are measured with approximately twice less points. Note than reducing by a factor of 2 the resolution in $1 \mathrm{D}$ allows the computation to run approximately four times faster since twice less points are used and time-step is twice bigger (cf. required cpu times on figure $7 \mathrm{~b}$ ). In $3 \mathrm{D}$, computation would be 16 times faster if twice less points are used in each direction. No major differences of stability and accuracy are observed between the implicit and the explicit hybrid schemes. However, the explicit colocated scheme requires a higher resolution than the implicit scheme. Apparently, the hybrid explicit scheme is the most efficient to solve this 1D laminar flame and presents some practical advantage compared to the colocated strategy when lower limit resolution is required to reduce $\mathrm{CPU}$ cost.

\subsection{Multidimensional extensions}

The present hybrid numerical treatment of BCs can also be implemented in several space dimensions. DNS tests like planar jets, 2D exiting vortices, decaying homogeneous turbulence, Poiseuille flow, have been performed and validated. The hybrid explicit approach always appears to be the best choice for reducing CPU cost while preserving pertinent solution. Increased stability balances the additive cost needed to interpolate variables from one grid to another. From a practical point of view, explicit finite difference scheme seems as stable and accurate as implicit. This significantly simplifies the parallelization procedure by avoiding Thomas algorithm [19].

To test multidimensional boundary treatment, a pre-computed turbulence is injected from the left of the domain though a non-reflecting inlet and ignited in the middle of the domain by a Gaussian temperature profile. Computational domain is a $2 \times 4 \mathrm{~cm}^{2}$ rectangle; turbulence intensity 
is $U^{\prime} / S_{L} \simeq 1$ and integral length scale $L_{i}=3 \mathrm{~mm}$. The flow is injected with a mean velocity $U^{i n} / S_{L}=1.62$. The injection is carried out using Taylor hypothesis $\left(\partial / \partial t=U^{i n} \cdot \partial / \partial x\right)$ to specify ingoing characteristic wave with a subsonic non-reflecting inlet. This initialization gives birth to two flame fronts (see figure 9a). The right one is blown through the right outflow while the left front tends to go up to the left inlet. Compared to the 1D situation, this test is even more discriminating in the sense that transverse, diffusive and reactive terms have to be taken into account to make the flame correctly exit the simulation (cf. [13]). Boundary conditions detailed analysis shall be discussed elsewhere [27], but figure 9 shows correct behaviour of $\mathrm{BC}$ treatment for the reactive front.

(a)

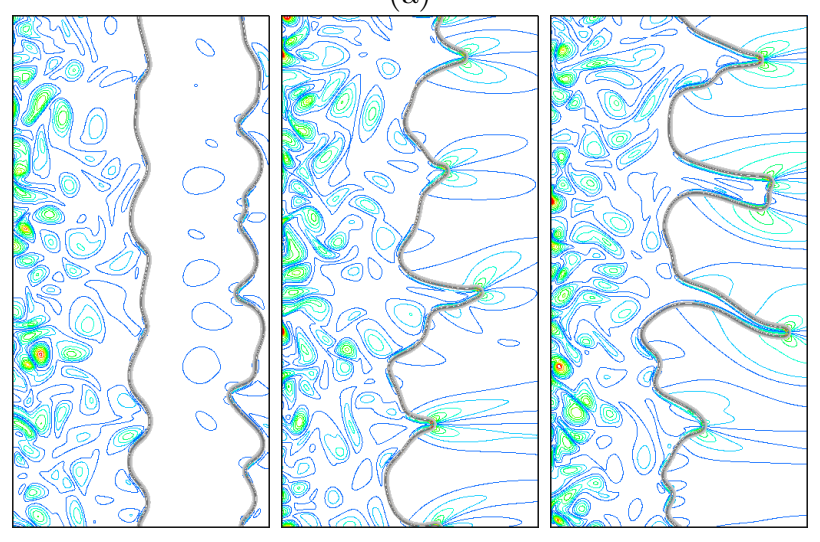

(b)

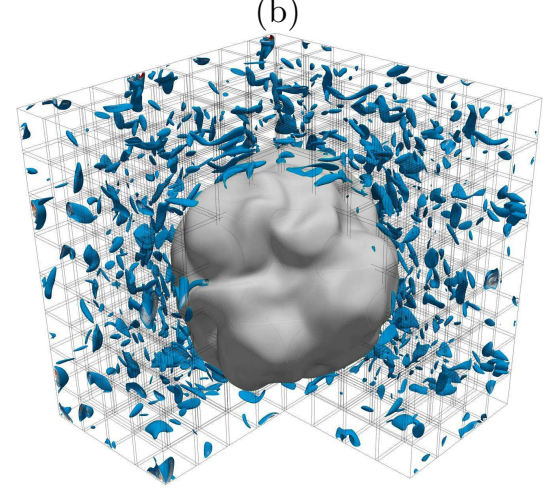

Figure 9: Simulation of turbulent stoichiometric propane-air expanding flames with $6^{\text {th }}$ order finite difference schemes with a hybrid arrangement of variables. Representation of iso-contours of vorticity (coloured) and iso-contours of reaction rate (gray). (a) propagation of two flame fronts with injection of turbulence. (b) Parallel simulation of a spherical flame expanding in a $(3 \mathrm{~cm})^{3}$ $(512 \times 4.7 G H z)$.

The presented $6^{t h}$ order hybrid explicit scheme can be parallelized, allowing large scale computations.

Three neighbor layers have to be transferred at each differentiation or interpolation step. Figure $9 \mathrm{~b}$ shows a sample result obtained with the parallel version of the code : a stoichiometric propaneair expanding flame computed using 512 processors at $4.7 \mathrm{GHz}$ with single step chemistry. The computational domain is $(3 \mathrm{~cm})^{3}$, discretized by $480^{3}$ points. Initial turbulent field intensity is $U^{\prime} / S_{L}=0.85$ and integral length scale is $L_{i}=3 \mathrm{~mm}$. This parallelization has an efficiency of $80 \%$ defined relatively to the sequential code. Non-reflecting outflows of Yoo et Im [13] are used on each faces in order to get free expansion of the flame. No particular problems were encountered for edge or corner treatments. Results of these computations will be analyzed elsewhere.

Note that colocated simulations (see e.g. [7] or [3]) would have required much more CPU time. For an explicit colocated simulation, the minimal resolution, imposed both from stability and accuracy requirements, is above 250 points per $\mathrm{cm}$ (mesh size of $40 \mu \mathrm{m}$ ), to be compared to 160 points per $\mathrm{cm}$ (mesh size of $62.5 \mu \mathrm{m}$ ). So CPU cost is divided by $6 \simeq(250 / 160)^{4}$. With an implicit compact scheme as used in [7], cpu-time would be higher because of the inversion cost of linear systems. For implicit (Padé) schemes, parallelization efficiency decrease (due to Thomas algorithm) 
would also significantly increase effective CPU cost.

\section{CONCLUSION.}

A hybrid colocated-staggered grid arrangement has been presented. Different grids, interpolation and differentiation finite difference operators have been introduced and clearly identified. The hybrid approach conciliates both the stability advantage of staggered low dissipative schemes with the unambiguous definition of boundaries in colocated arrangement, allowing the use of characteristic boundary conditions treatment. Fourier analysis shows increased numerical accuracy not only at boundaries but also on the overall computational solution. The analysis is illustrated by $1 \mathrm{D}, 2 \mathrm{D}$ and 3D compressible reactive or non reactive flow simulations. Hybrid schemes need less grid points to obtain numerical pertinent results and can be easily coupled with improved 3D Navier-Stokes Characteristic Boundary Conditions on multidimensional reactive simulations. This explicit hybrid strategy is particularly interesting when lower limit resolutions have to be used to compute a large multidimensional problem at reduced CPU cost, like the expanding flame shown previous section.

\section{TAYLOR EXPANSION METHODOLOGY.}

Finite difference formulas are commonly used and a large amount of discrete differential expressions are available in the literature $[16,6]$. Weighting coefficients of a finite difference formula depend on grid arrangement, used stencil and formal accuracy. In [6], FD formulas for colocated stencils are established (by matching Taylor series coefficients). For first and second derivative, Dvornikov [22] shows how to compute coefficients for uniform symmetrical stencils of any even order. Li [23] then extended the method in 2005 for general stencils (i.e. not necessary symmetrical nor uniform). This iterative method allows to compute coefficients for any $n^{\text {th }}$ derivative at any order $N$, but is not valid for implicit schemes. Shukla et Zhong [24] derive implicit finite-difference formulas at any order of accuracy, for first and second derivative. However, generality of the obtained formulas is balanced out by intricate expressions depending on Lagrange polynomials and their derivatives.

For the present hybrid arrangement, weighting coefficients have been determined by using Taylor expansion matching. Coefficients $\left(\bar{\alpha}_{0}, \ldots, \bar{\alpha}_{N_{\alpha}}, \bar{\beta}_{1}, \ldots, \bar{\beta}_{N_{\beta}}\right)$ of the interpolation formula (5a) are determined by system (9). Note that these coefficients depend only on the shape of the stencil $a_{1}, \ldots, a_{N_{\alpha}}, b_{1}, \ldots, b_{N_{\beta}}$, where $a_{k}$ and $b_{k}$ are integers or half-integers.

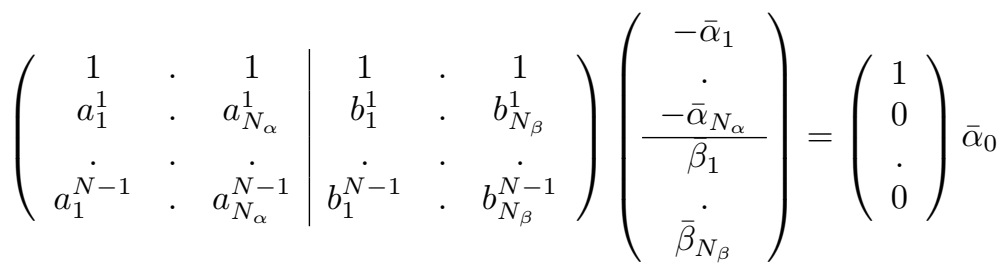

If $f$ is a sufficiently smooth function, its Taylor expansion writes $f(X)=c_{0}+\sum_{k=1}^{N-1} c_{k} X^{k}+$ $\mathcal{O}\left(X^{N}\right)$, with $c_{k} \in \mathbb{R}$ for $k \in[0 ; N-1]$. Replacing $\bar{f}_{n+a_{k}}$ and $f_{n+b_{k}}$ in relation (5a) $\left(X=x-x\left(n_{i}\right)=\right.$ $a_{k} \Delta x$ or $X=b_{k} \Delta x$ ), and in order to verify relation (5a) for any set of $c_{k}$ coefficients, system (9) is obtained. Left-hand side matrix of this system is a Vandermonde matrix of size $N \times N$ with 
$N=N_{\alpha}+N_{\beta}$ (and the system is invertible as soon as the $a_{k}$ and $b_{k}$ are distinct one another). Formal accuracy is linked to the number of points used in the stencil.

In a similar way, weighting coefficients $\left(\dot{\alpha}_{0}, \ldots, \dot{\alpha}_{N_{\alpha}}, \dot{\beta}_{1}, \ldots, \dot{\beta}_{N_{\beta}+1}\right)$ of the first derivative formula (5b) are determined by system (10) for a formal accuracy of order $N$. These coefficients depend on the stencil arrangement $\left(a_{1}, \ldots, a_{N_{\alpha}}, b_{1}, \ldots, b_{N_{\beta}+1}\right)$. When the stencil used in relations (5a) and (5b) is centered $\left(a_{2}=-a_{1}, b_{2}=-b_{1}, b_{4}=-b_{3}, \ldots\right)$, note that systems (9) and (10) present symmetries, which implies symmetrical weighting coefficients $\left(\bar{\alpha}_{2}=-\bar{\alpha}_{1}, \bar{\beta}_{2}=\bar{\beta}_{1}, \bar{\beta}_{4}=\bar{\beta}_{3}\right.$, and $\dot{\alpha}_{2}=-\dot{\alpha}_{1}$, $\left.\dot{\beta}_{2}=-\dot{\beta}_{1}, \dot{\beta}_{4}=-\dot{\beta}_{3}\right)$.

$$
\left(\begin{array}{ccc|ccc}
0 & \cdot & 0 & 1 & \cdot & 1 \\
1 & \cdot & 1 & b_{1}^{1} & \cdot & b_{N_{\beta}+1}^{1} \\
2 . a_{1}^{1} & \cdot & 2 . a_{N_{\alpha}}^{1} & b_{1}^{2} & \cdot & b_{N_{\beta}+1}^{2} \\
\cdot & \cdot & \cdot & \cdot & \cdot & \cdot \\
N a_{1}^{N-1} & \cdot & N a_{N_{\alpha}}^{N-1} & b_{1}^{N} & \cdot & b_{N_{\beta}+1}^{N}
\end{array}\right)\left(\begin{array}{c}
-\dot{\alpha}_{1} \\
\cdot \\
-\dot{\alpha}_{N_{\alpha}} \\
\hline \dot{\beta}_{1} \\
\cdot \\
\dot{\beta}_{N_{\beta}+1}
\end{array}\right)=\left(\begin{array}{c}
0 \\
1 \\
0 \\
\cdot \\
0
\end{array}\right) \dot{\alpha}_{0}
$$

As for the interpolation, function $f$ is Taylor expanded as $f(X)=c_{0}+\sum_{k=1}^{N} c_{k} X^{k}+\mathcal{O}\left(X^{N+1}\right)$. For the first derivative $\dot{f}$, the Taylor series is written as $\dot{f}(X)=c_{1}+\sum_{k=2}^{N} k c_{k} X^{k-1}+\mathcal{O}\left(X^{N}\right)$. Substituting $\dot{f}_{n+a_{k}}$ and $f_{n+b_{k}}$ by their expanded expressions in relation (5b), system (10) is then obtained, since relation (5b) must be verified for any set of $c_{k}$ coefficients. This system can conveniently be inverted using for instance symbolic computation software. Simple expressions for weighting coefficients of explicit schemes can be derived :

$$
\begin{array}{lll}
\bar{f}_{n}=\sum_{k=1}^{N} \bar{\beta}_{k} \cdot f_{n+b_{k}}+\mathcal{O}\left(\Delta x^{N}\right) & \text { with } & \bar{\beta}_{k}=\prod_{\substack{j=1 \\
j \neq k}}^{N} \frac{b_{j}}{b_{j}-b_{k}} \\
\dot{f}_{n}=\sum_{k=1}^{N} \dot{\beta}_{k} \cdot \frac{f_{n+b_{k}}-f_{n}}{\Delta x}+\mathcal{O}\left(\Delta x^{N}\right) & \text { with } & \dot{\beta}_{k}=\frac{\bar{\beta}_{k}}{b_{k}} \\
\dot{f}_{n}=\sum_{k=1}^{N} \dot{\beta}_{k} \cdot \frac{f_{n+b_{k}}}{\Delta x}+\mathcal{O}\left(\Delta x^{N-1}\right) & \text { with } & \dot{\beta}_{k}=\bar{\beta}_{k}\left(\frac{1}{b_{k}}-\sum_{l=1}^{N+1} \frac{\bar{\beta}_{l}}{b_{l}}\right)
\end{array}
$$

Equation (11) is a finite difference formula for explicit interpolation at order $N$. Expressions (12) and (13) are explicit finite difference formulas for first-order differentiation.

For colocated arrangement, first derivative finite-difference schemes (analysed in the present work) are given table 5. Implicit and explicit formulas can respectively be obtained by relations (10) and (12). Formal order of accuracy is decreased at boundary to keep the same stencil width at inner and boundary points. On each side, this stencil width is $3 \Delta x$ for the explicit formulation versus $2 \Delta x$ for the compact implicit scheme.

Most staggered finite-difference formulas are available in literature and the presently used formulas are recalled table 6 . At the boundaries, formal accuracy order decreases to keep stencil width constant. For $6^{\text {th }}$ order accuracy (for inner points), stencil width is $5 \Delta x / 2$ on each side with the explicit formulation and $3 \Delta x / 2$ with the implicit formulation.

For a hybrid arrangement, finite-difference formulas are the same as staggered ones within the domain. They are modified at the boundaries. All these modified formulas can be derived from systems (9) and (10) for respectively interpolation and first-derivative operators. Again, expressions 
Table 5: First derivative finite difference schemes for a colocated arrangement.

\begin{tabular}{|c|c|c|c|c|c|}
\hline \multicolumn{2}{|c|}{ operator } & \multicolumn{2}{|c|}{ Finite Difference formulæ } & $N$ & refs \\
\hline \multirow{7}{*}{$\stackrel{\oplus}{\oplus}$} & \multirow{4}{*}{ 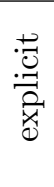 } & C_3e & $\dot{f}_{1}=-\frac{11}{6} \frac{f_{1}}{\Delta x}+3 \frac{f_{2}}{\Delta x}-\frac{3}{2} \frac{f_{3}}{\Delta x}+\frac{1}{3} \frac{f_{4}}{\Delta x}$ & 3 & [6] \\
\hline & & C_4e & $\dot{f}_{2}=-\frac{1}{4} \frac{f_{1}}{\Delta x}-\frac{5}{6} \frac{f_{2}}{\Delta x}+\frac{3}{2} \frac{f_{3}}{\Delta x}-\frac{1}{2} \frac{f_{4}}{\Delta x}+\frac{1}{12} \frac{f_{5}}{\Delta x}$ & 4 & {$[6]$} \\
\hline & & C_5e & $\dot{f}_{3}=+\frac{1}{20} \frac{f_{1}}{\Delta x}-\frac{1}{2} \frac{f_{2}}{\Delta x}-\frac{1}{3} \frac{f_{3}}{\Delta x}+\frac{f_{4}}{\Delta x}-\frac{1}{4} \frac{f_{5}}{\Delta x}+\frac{1}{30} \frac{f_{6}}{\Delta x}$ & 5 & {$[6]$} \\
\hline & & C_6e & $\dot{f}_{n}=+\frac{3}{4} \frac{f_{n+1}-f_{n-1}}{\Delta x}-\frac{3}{20} \frac{f_{n+2}-f_{n-2}}{\Delta x}+\frac{1}{60} \frac{f_{n+3}-f_{n-3}}{\Delta x}$ & 6 & {$[6]$} \\
\hline & \multirow{3}{*}{ 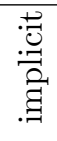 } & C_3i & $2 \dot{f}_{1}+4 f_{2}=-5 \frac{f_{1}}{\Delta x}+4 \frac{f_{2}}{\Delta x}+\frac{f_{3}}{\Delta x}$ & 3 & [6] \\
\hline & & C_4i & $\dot{f}_{n-1}+4 \dot{f}_{n}+\dot{f}_{n+1}=3 \frac{\vec{f}_{n+1}-\bar{f}_{n-1}}{\Delta x}$ & 4 & {$[6]$} \\
\hline & & C_6i & $\dot{f}_{n-1}+3 \dot{f}_{n}+\dot{f}_{n+1}=\frac{7}{3} \frac{f_{n+1}-f_{n-1}}{\Delta x}+\frac{1}{12} \frac{f_{n+2}-f_{n-2}}{\Delta x}$ & 6 & {$[6]$} \\
\hline
\end{tabular}

are chosen in order to keep a constant stencil range all over the domain and to insure a $6^{\text {th }}$ order formal accuracy at inner points.

\section{Acknowledgments}

This work was granted access to the HPC resources of CRIHAN and also IDRIS under the allocation 2010-026186 made by GENCI (Grand Equipement National de Calcul Intensif). Authors have benefited from fruitful interactions with Dr. G. Fernandez from PARALGO and with participants of the May 2010 Nordita Program on Turbulent Combustion in Stockholm.

\section{References}

[1] Ferziger JH, Peric M, Morton KW. Computational methods for fluid dynamics. Springer Berlin, 1999.

[2] Harlow FH, Welch JE. Numerical Calculation of Time-Dependent Viscous Incompressible Flow of Fluid with Free Surface. Physics of Fluids,1965.

[3] Babkovskaia N, Haugen NEL, Brandenburg A. A high-order public domain code for direct numerical simulations of turbulent combustion arXiv:1005.5301v1 [astro-ph.SR], may 28th 2010.

[4] Thompson KW. Time-dependent boundary conditions for hyperbolic systems I. Journal of Computational Physics, 1987.

[5] Vervisch L, Labegorre B, Réveillon J. Hydrogen-sulphur oxy-flame analysis and single-step flame tabulated chemistry. Fuel, Elsevier, 2004.

[6] Lele S. Compact finite difference schemes with spectral-like resolution. Journal of Computational Physics, 1992.

[7] Van Oijen JA, Bastiaans RJM, Groot GRA \& L.P.H. De Goey LPH. Direct Numerical Simulations of Premixed Turbulent Flames with Reduced Chemistry: Validation and Flamelet Analysis Flow, Turbulence and Combustion 75: 67-84, 2005. 
Table 6: Finite difference schemes for differentiation and interpolation for a staggered arrangement. ("PW" stands for "Present Work")

\begin{tabular}{|c|c|c|c|c|c|}
\hline \multicolumn{2}{|c|}{ operator } & \multicolumn{2}{|c|}{ Finite Difference formulæ } & \multirow[t]{2}{*}{$N$} & \multirow{2}{*}{$\begin{array}{l}\text { refs } \\
\text { PW }\end{array}$} \\
\hline \multirow{7}{*}{$\underset{\oplus}{\rightleftarrows}$} & \multirow{4}{*}{ 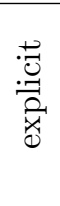 } & Sd1_2e & $\dot{f}_{1}=-2 \frac{f_{3 / 2}}{\Delta x}+3 \frac{f_{5 / 2}}{\Delta x}-\frac{f_{7 / 2}}{\Delta x}$ & & \\
\hline & & Sd1_3e & $\dot{f}_{2}=-\frac{23}{24} \frac{f_{3 / 2}}{\Delta x}+\frac{7}{8} \frac{f_{5 / 2}}{\Delta x}+\frac{1}{8} \frac{f_{7 / 2}}{\Delta x}-\frac{1}{24} \frac{f_{9 / 2}}{\Delta x}$ & 3 & {$[8]$} \\
\hline & & Sd1_4e & $\dot{f}_{3}=\frac{9}{8} \frac{f_{7 / 2}-f_{5 / 2}}{\Delta x}-\frac{1}{24} \frac{f_{9 / 2}-f_{3 / 2}}{\Delta x}$ & 4 & {$[6,8]$} \\
\hline & & Sd1_6e & $\dot{f}_{n}=\frac{75\left(f_{n+1 / 2}-f_{n-1 / 2}\right)}{64 \Delta x}-\frac{25\left(f_{n+3 / 2}-f_{n-3 / 2}\right)}{384 \Delta x}+\frac{3\left(f_{n+5 / 2}-f_{n-5 / 2}\right)}{640 \Delta x}$ & 6 & {$[6]$} \\
\hline & \multirow{3}{*}{ 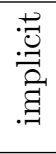 } & Sd1_3i & $\dot{f}_{1}+23 \dot{f}_{2}=-25 \frac{f_{3 / 2}}{\Delta x}+26 \frac{f_{5 / 2}}{\Delta x}-\frac{f_{7 / 2}}{\Delta x}$ & 3 & {$[9,10]$} \\
\hline & & Sd1_4i & $\dot{f}_{n-1}+22 \dot{f}_{n}+\dot{f}_{n+1}=24 \frac{f_{n+1 / 2}-f_{n-1 / 2}}{\Delta x}$ & 4 & {$[6]$} \\
\hline & & Sd1_6i & $\dot{f}_{n-1}+\frac{62}{9} \dot{f}_{n}+\dot{f}_{n+1}=7 \frac{f_{n+1 / 2}-\dot{f}_{n-1 / 2}}{\Delta x}+\frac{17}{27} \frac{f_{n+3 / 2}-f_{n-3 / 2}}{\Delta x}$ & 6 & {$[6]$} \\
\hline \multirow{7}{*}{ 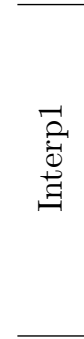 } & \multirow{4}{*}{ 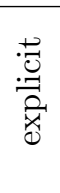 } & Si1_3e & $\bar{f}_{1}=\frac{15}{8} f_{3 / 2}-\frac{5}{4} f_{5 / 2}+\frac{3}{8} f_{7 / 2}$ & 3 & {$[9,10]$} \\
\hline & & Si1_4e & $\bar{f}_{2}=\frac{5}{16} f_{3 / 2}+\frac{15}{16} f_{5 / 2}-\frac{5}{16} f_{7 / 2}+\frac{1}{16} f_{9 / 2}$ & 4 & {$[8,9]$} \\
\hline & & Si1_5e & $\bar{f}_{3}=-\frac{5}{128} f_{3 / 2}+\frac{15}{32} f_{5 / 2}+\frac{45}{64} f_{7 / 2}-\frac{5}{32} f_{9 / 2}+\frac{3}{128} f_{11 / 2}$ & 5 & PW \\
\hline & & Si1_6e & $\bar{f}_{n}=\frac{75\left(f_{n+1 / 2}+f_{n-1 / 2}\right)}{128}-\frac{25\left(f_{n+3 / 2}+f_{n-3 / 2}\right)}{256}+\frac{3\left(f_{n+5 / 2}+f_{n-5 / 2}\right)}{256}$ & 6 & {$[6]$} \\
\hline & \multirow{3}{*}{ 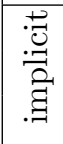 } & Si1_3i & $\bar{f}_{1}+3 \bar{f}_{2}=3 f_{3 / 2}+f_{5 / 2}$ & 3 & $\sim[9]$ \\
\hline & & Si1_4i & $\bar{f}_{n-1}+6 \bar{f}_{n}+\bar{f}_{n+1}=4\left(f_{n+1 / 2}+f_{n-1 / 2}\right)$ & 4 & {$[6]$} \\
\hline & & Si1_6i & $\bar{f}_{n-1}+\frac{10}{3} \bar{f}_{n}+\bar{f}_{n+1}=\frac{5\left(f_{n+1 / 2}+f_{n-1 / 2}\right)}{2}+\frac{f_{n+3 / 2}+f_{n-3 / 2}}{6}$ & 6 & {$[6]$} \\
\hline \multirow{6}{*}{$\begin{array}{l}\mathscr{H} \\
\ddot{\theta}\end{array}$} & \multirow{3}{*}{ 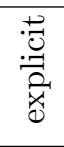 } & Sd2_3e & $\dot{f}_{3 / 2}=-\frac{23}{24} \frac{f_{1}}{\Delta x}+\frac{7}{8} \frac{f_{2}}{\Delta x}+\frac{1}{8} \frac{f_{3}}{\Delta x}-\frac{1}{24} \frac{f_{4}}{\Delta x}$ & 3 & [8] \\
\hline & & $\mathrm{Sd} 2 \_4 \mathrm{e}$ & $\dot{f}_{5 / 2}=\frac{9}{8} \frac{f_{3}-f_{2}}{\Delta x}-\frac{1}{24} \frac{1}{\Delta x}-f_{1}$ & 4 & [6] \\
\hline & & Sd2_6e & $\dot{f}_{n+1 / 2}=+\frac{75}{64} \frac{f_{n+1}-f_{n}}{\Delta x}-\frac{25}{384} \frac{f_{n+2}-f_{n-1}}{\Delta x}+\frac{3}{640} \frac{f_{n+3}-f_{n-2}}{\Delta x}$ & 6 & {$[6]$} \\
\hline & \multirow{3}{*}{ 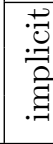 } & Sd2_3i & $\dot{f}_{3 / 2}+\dot{f}_{5 / 2}=\frac{f_{2}-f_{1}}{\Delta x}+\frac{f_{2}-f_{3}}{\Delta x}$ & 3 & PW \\
\hline & & $\mathrm{Sd} 2 \_4 \mathrm{i}$ & $\dot{f}_{n-1 / 2}+22 \dot{f}_{n+1 / 2}+\dot{f}_{n+3 / 2}=24 \frac{f_{n+1}-f_{n}}{\Delta x}$ & 4 & {$[6]$} \\
\hline & & Sd2_6i & $\dot{f}_{n-1 / 2}+\frac{62}{9} \dot{f}_{n+1 / 2}+\dot{f}_{n+3 / 2}=7 \frac{f_{n+1}-f_{n}}{\Delta x}+\frac{17}{27} \frac{f_{n+2}-f_{n-1}}{\Delta x}$ & 6 & {$[6]$} \\
\hline \multirow{6}{*}{ 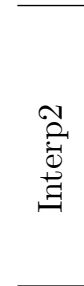 } & \multirow{3}{*}{ 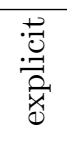 } & Si2_4e & $\bar{f}_{3 / 2}=\frac{5}{16} f_{1}+\frac{15}{16} f_{2}-\frac{5}{16} f_{3}+\frac{1}{16} f_{4}$ & 4 & {$[8,9]$} \\
\hline & & Si2_5e & $\bar{f}_{5 / 2}=-\frac{5}{128} f_{1}+\frac{15}{32} f_{2}+\frac{45}{64} f_{3}-\frac{5}{32} f_{4}+\frac{3}{128} f_{5}$ & 5 & $\mathrm{PW}$ \\
\hline & & Si2_6e & $\bar{f}_{n+1 / 2}=\frac{75\left(f_{n+1}+f_{n}\right)}{128}-\frac{25\left(f_{n+2}+f_{n-1}\right)}{256}+\frac{3\left(f_{n+3}+f_{n-2}\right)}{256}$ & 6 & {$[6]$} \\
\hline & \multirow{3}{*}{ 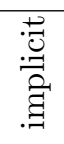 } & Si2_4i & $\bar{f}_{3 / 2}+\bar{f}_{5 / 2}=\frac{1}{4} f_{1}+\frac{3}{2} f_{2}+\frac{1}{4} f_{3}$ & 4 & $\sim[9]$ \\
\hline & & Si2_5i & $\bar{f}_{n-1 / 2}+6 \bar{f}_{n+1 / 2}+\bar{f}_{n+3 / 2}=4\left(f_{n+1}-f_{n}\right)$ & 4 & {$[6]$} \\
\hline & & Si2_6i & $\bar{f}_{n-1 / 2}+\frac{10}{3} \bar{f}_{n+1 / 2}+\bar{f}_{n+3 / 2}=\frac{5\left(f_{n+1}-f_{n}\right)}{2}+\frac{f_{n+2}-f_{n-1}}{6}$ & 6 & {$[6]$} \\
\hline
\end{tabular}

[8] Yefet A, Petropoulos PG. A Non-dissipative Staggered Fourth-order Accurate Explicit Finite Difference Scheme for the Time-domain Maxwell's Equations. NASA, Langley Research Center; National Technical Information Service, distributor,1999.

[9] Nagarajan S, Lele SK, Ferziger JH. A robust high-order compact method for large eddy simulation. Journal of Computational Physics, 2003.

[10] Boersma BJ. A staggered compact finite difference formulation for the compressible NavierStokes equations. Journal of Computational Physics, 2005. 
[11] Boersma BJ, Hoeven SV. Low dissipative methods for turbulent reacting flows. European Conference on CFD, 2006.

[12] Poinsot TJ, Lele SK. Boundary conditions for direct simulations of compressible viscous flows. Journal of computational physics, 1992.

[13] Yoo CS, Im HG. Characteristic boundary conditions for simulations of compressible reacting

Table 7: Finite difference schemes for differentiation and interpolation for a hybrid arrangement ("PW" stands for "Present Work").

\begin{tabular}{|c|c|c|c|c|c|}
\hline \multicolumn{2}{|c|}{ operator } & \multicolumn{2}{|c|}{ Finite Difference formulæ } & \multirow{2}{*}{$\begin{array}{c}N \\
3 \\
\end{array}$} & \multirow{2}{*}{$\begin{array}{l}\text { refs } \\
\text { PW }\end{array}$} \\
\hline \multirow{7}{*}{$\underset{\oplus}{\rightleftarrows}$} & \multirow{4}{*}{ 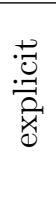 } & Hd1_3e & $\dot{f}_{1}=-\frac{46}{15} \frac{f_{1}}{\Delta x}+\frac{15}{4} \frac{f_{3 / 2}}{\Delta x}-\frac{5}{6} \frac{f_{5 / 2}}{\Delta x}+\frac{3}{20} \frac{f_{7 / 2}}{\Delta x}$ & & \\
\hline & & $\mathrm{Hd} 1 \_4 \mathrm{e}$ & $\dot{f}_{2}=\frac{16}{105} \frac{f_{1}}{\Delta x}-\frac{31}{24} \frac{f_{3 / 2}}{\Delta x}+\frac{29}{24} \frac{f_{5 / 2}}{\Delta x}-\frac{3}{40} \frac{f_{7 / 2}}{\Delta x}+\frac{1}{168} \frac{f_{9 / 2}}{\Delta x}$ & 4 & PW \\
\hline & & Hd1_5e & $\dot{f}_{3}=-\frac{2 f_{1}}{105 \Delta x}+\frac{17 f_{3 / 2}}{192 \Delta x}-\frac{19 f_{5 / 2}}{16 \Delta x}+\frac{189 f_{7 / 2}}{160 \Delta x}-\frac{23 f_{9 / 2}}{336 \Delta x}+\frac{f_{11 / 2}}{192 \Delta x}$ & 5 & $\mathrm{PW}$ \\
\hline & & Hd1_6e & $\dot{f}_{n}=+\frac{75}{64} \frac{f_{n+1 / 2}-f_{n-1 / 2}}{\Delta x}-\frac{25}{384} \frac{f_{n+3 / 2}-f_{n-3 / 2}}{\Delta x}+\frac{3}{640} \frac{f_{n+5 / 2}-f_{n-5 / 2}}{\Delta x}$ & 6 & {$[6]$} \\
\hline & \multirow{3}{*}{ 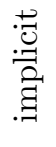 } & Hd1_3i & $\dot{f}_{1}+3 \dot{f}_{2}=\frac{8}{3} \frac{f_{3 / 2}-f_{1}}{\Delta x}$ & 3 & PW \\
\hline & & Hd1_4i & $\dot{f}_{n-1}+22 \dot{f}_{n}+\dot{f}_{n+1}=24 \frac{f_{n+1 / 2}-f_{n-1 / 2}}{\Delta x}$ & 4 & {$[6]$} \\
\hline & & Hd1_6i & $\dot{f}_{n-1}+\frac{62}{9} \dot{f}_{n}+\dot{f}_{n+1}=7 \frac{f_{n+1 / 2}-\dot{f}_{n-1 / 2}}{\Delta x}+\frac{17}{27} \frac{f_{n+3 / 2}-f_{n-3 / 2}}{\Delta x}$ & 6 & {$[6]$} \\
\hline \multirow{7}{*}{$\begin{array}{l}\overrightarrow{\overrightarrow{0}} \\
\overrightarrow{0} \\
\stackrel{\Xi}{\Xi}\end{array}$} & \multirow{4}{*}{ 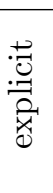 } & Hi1_0e & $\bar{f}_{1}=f_{1}$ & $\infty$ & PW \\
\hline & & Hi1_4e & $\bar{f}_{2}=-\frac{1}{7} f_{1}+\frac{5}{8} f_{3 / 2}+\frac{5}{8} f_{5 / 2}-\frac{1}{8} f_{7 / 2}+\frac{1}{56} f_{9 / 2}$ & 5 & $\mathrm{PW}$ \\
\hline & & Hi1_5e & $\bar{f}_{3}=\frac{1}{21} f_{1}-\frac{5}{32} f_{3 / 2}+\frac{5}{8} f_{5 / 2}+\frac{9}{16} f_{7 / 2}-\frac{5}{56} f_{9 / 2}+\frac{1}{96} f_{11 / 2}$ & 6 & PW \\
\hline & & Hi1_6e & $\bar{f}_{n}=\frac{\overline{75}\left(f_{n+1 / 2}+f_{n-1 / 2}\right)}{128}-\frac{25\left(f_{n+3 / 2}+f_{n-3 / 2}\right)}{256}+\frac{3\left(f_{n+5 / 2}+f_{n-5 / 2}\right)}{256}$ & 6 & {$[6]$} \\
\hline & \multirow{3}{*}{ 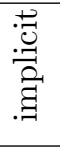 } & Hi1_0i & $\bar{f}_{1}=f_{1}$ & $\infty$ & $\mathrm{PW}$ \\
\hline & & Hi1_4i & $f_{n-1}+6 f_{n}+f_{n+1}=4\left(f_{n+1 / 2}+f_{n-1 / 2}\right)$ & 4 & {$[6]$} \\
\hline & & Hi1_6i & $\bar{f}_{n-1}+\frac{10}{3} \bar{f}_{n}+\bar{f}_{n+1}=\frac{5\left(f_{n+1 / 2}+f_{n-1 / 2}\right)}{2}+\frac{f_{n+3 / 2}+f_{n-3 / 2}}{6}$ & 6 & {$[6]$} \\
\hline \multirow{7}{*}{$\stackrel{\mathscr{\varphi}}{\mathscr{\varphi}}$} & \multirow{4}{*}{ 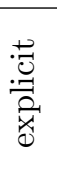 } & $\mathrm{Hd} 2 \_3 \mathrm{e}$ & $\dot{f}_{1}=-\frac{11}{6} \frac{f_{1}}{\Delta x}+3 \frac{f_{2}}{\Delta x}-\frac{3}{2} \frac{f_{3}}{\Delta x}+\frac{1}{3} \frac{f_{4}}{\Delta x}$ & 3 & {$[6]$} \\
\hline & & $\mathrm{Hd} 2 \_4 \mathrm{e}$ & $f_{3 / 2}=-\frac{23}{24} \frac{f_{1}}{\Delta x}+\frac{7}{8} \frac{f_{2}}{\Delta x}+\frac{1}{8} \frac{f_{3}}{\Delta x}-\frac{1}{24} \frac{f_{4}}{\Delta x}$ & 3 & {$[8]$} \\
\hline & & $\mathrm{Hd} 2 \_5 \mathrm{e}$ & $f_{5 / 2}=\frac{9}{8} \frac{f_{3}-f_{2}}{\Delta x}-\frac{1}{24} \frac{f_{4}-f_{1}}{\Delta x}$ & 4 & {$[6,8]$} \\
\hline & & $\mathrm{Hd} 2 \_6 \mathrm{e}$ & $\dot{f}_{n+1 / 2}=+\frac{75}{64} \frac{f_{n+1}-f_{n}}{\Delta x}-\frac{25}{384} \frac{f_{n+2}-f_{n-1}}{\Delta x}+\frac{3}{640} \frac{f_{n+3}-f_{n-2}}{\Delta x}$ & 6 & {$[6]$} \\
\hline & \multirow{3}{*}{ 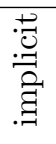 } & $\mathrm{Hd} 2 \_3 \mathrm{i}$ & $\frac{1}{8} \dot{f}_{1}+\dot{f}_{3 / 2}=-\frac{19}{16} \frac{f_{1}}{\Delta x}+\frac{5}{4} \frac{f_{2}}{\Delta x}-\frac{1}{16} \frac{f_{3}}{\Delta x}$ & 3 & PW \\
\hline & & $\mathrm{Hd} 2 \_4 \mathrm{i}$ & $\dot{f}_{1}+6 f_{3 / 2}+2 f_{5 / 2}=-\frac{15}{2} \frac{f_{1}}{\Delta x}+6 \frac{f_{2}}{\Delta x}+\frac{3}{2} \frac{f_{3}}{\Delta x}$ & 4 & PW \\
\hline & & Hd2_6i & $\dot{f}_{n-1 / 2}+\frac{62}{9} \dot{f}_{n+1 / 2}+\dot{f}_{n+3 / 2}=7 \frac{f_{n+1}-f_{n}}{\Delta x}+\frac{17}{27} \frac{f_{n+2}-f_{n-1}}{\Delta x}$ & 6 & {$[6]$} \\
\hline \multirow{7}{*}{ 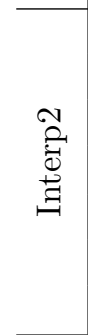 } & \multirow{4}{*}{ 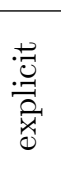 } & Hi2_0e & $\bar{f}_{1}=f_{1}$ & $\infty$ & PW \\
\hline & & Hi2_4e & $\bar{f}_{3 / 2}=\frac{5}{16} f_{1}+\frac{15}{16} f_{2}-\frac{5}{16} f_{3}+\frac{1}{16} f_{4}$ & 4 & {$[8,9]$} \\
\hline & & Hi2_5e & $\bar{f}_{5 / 2}=-\frac{5}{128} f_{1}+\frac{15}{32} f_{2}+\frac{45}{64} f_{3}-\frac{5}{32} f_{4}+\frac{3}{128} f_{5}$ & 5 & $\mathrm{PW}$ \\
\hline & & Hi2_6e & $\bar{f}_{n+1 / 2}=\frac{75\left(f_{n+1}+f_{n}\right)}{128}-\frac{25\left(f_{n+2}+f_{n-1}\right)}{128}+\frac{3\left(f_{n+3}+f_{n-2}\right)}{256}$ & 6 & {$[6]$} \\
\hline & \multirow{3}{*}{ 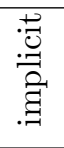 } & Hi2_0i & $f_{1}=f_{1}$ & $\infty$ & $\mathrm{PW}$ \\
\hline & & Hi2_4i & $\bar{f}_{1}+\bar{f}_{3 / 2}+\bar{f}_{5 / 2}=\frac{5}{4} f_{1}+\frac{3}{2} f_{2}+\frac{1}{4} f_{3}$ & 4 & $\mathrm{PW}$ \\
\hline & & Hi2_6i & $\bar{f}_{n-1 / 2}+\frac{10}{3} \bar{f}_{n+1 / 2}+\bar{f}_{n+3 / 2}=\frac{5\left(f_{n+1}-f_{n}\right)}{2}+\frac{f_{n+2}-f_{n-1}}{6}$ & 6 & {$[6]$} \\
\hline
\end{tabular}


flows with multi-dimensional, viscous and reaction effects. Combustion Theory and Modelling, 2007.

[14] Lodato G, Domingo P, Vervisch L. Three-dimensional boundary conditions for direct and largeeddy simulation of compressible viscous flows. Journal of Computational Physics, 2008.

[15] Yarrow M. Solving periodic block tridiagonal systems using Sherman Morrison Woodbury formula. AIAA Computational Fluid Dynamics conference, Buffalo, N.Y., 1989.

[16] Abramowitz M, Stegun IA. Handbook of Mathematical Functions with Formulas, Graphs, and Mathematical Table Courier Dover Publications, 1965.

[17] Carpenter MH, Gottlieb D, Abarbanel S. Stable and accurate boundary treatments for compact, high-order finite-difference schemes Applied Numerical Mathematics, 1993.

[18] Mahesh K. A family of high order finite difference schemes with good spectral resolution. Journal of Computational Physics,1998.

[19] Sun XH. Application and accuracy of the parallel diagonal dominant algorithm. Parallel Computing, 1995.

[20] Bogey C, Bailly C. A family of low dispersive and low dissipative explicit schemes for flow and noise computations. Journal of Computational Physics, 2004.

[21] Hirsch C, Numerical computation of internal and external flows: fundamentals of computational fluid dynamics. Elsevier/Butterworth-Heinemann, 2007.

[22] Dvornikov M. formulaef numerical differentiation. Arxiv preprint math/0306092, 2003.

[23] Li J. General explicit difference formulas for numerical differentiation. Journal of Computational and Applied Mathematics, 2005.

[24] Shukla RK, Zhong X. Derivation of high-order compact finite difference schemes for nonuniform grid using polynomial interpolation. Journal of Computational Physics, 2005.

[25] Topalian VD, Freund JB. Boundary Formulations for High-Order Finite Differences on Staggered Meshes. Computers \& Fluids, 2010.

[26] Albin E. Contribution à la modélisation numérique des flammes turbulentes : comparaisons DNS-EEM-Expériences. CORIA-INSA Rouen, PhD thesis report, april 27th 2010.

[27] Albin E, D'Angelo Y, Vervisch L. Flow streamline based Navier-Stokes Characteristic Boundary Conditions: modelling for transverse and corner outflows. Computers \& Fluids, submitted, 2010.

[28] Wray A. Minimal storage time-advancement schemes for spectral methods. Preprint, 1991. 